\title{
Ameliorating the popular lepton mixings with $A 4$ symmetry: A seesaw model for realistic neutrino masses and mixing
}

\author{
Soumita Pramanick ${ }^{*}$ \\ Harish-Chandra Research Institute, Chhatnag Road, Jhunsi, Allahabad 211019, India \\ and Department of Physics, University of Calcutta, \\ 92 Acharya Prafulla Chandra Road, Kolkata 700009, India
}

(Received 27 March 2018; published 17 October 2018)

\begin{abstract}
A model for neutrino masses and mixing is presented using the seesaw mechanism. The model combines type-I and type-II seesaw contributions of which the latter dominates. The scalars and the leptons in the model are assigned $A 4$ charges suitable to obtain the mass matrices required for the scheme. The type-II seesaw accommodates atmospheric mass splitting and maximal mixing in the atmospheric sector $\left(\theta_{23}=\pi / 4\right)$. It is characterized by vanishing solar mass splitting and $\theta_{13}$ whereas the third neutrino mixing angle can acquire any value, $\theta_{12}^{0}$. Particular alternatives of $\theta_{12}^{0}$ viz. $\theta_{12}^{0}=35.3^{\circ}$ (tribimaximal), $45.0^{\circ}$ (bimaximal), $31.7^{\circ}$ (golden ratio) are accounted for. Another choice of $\theta_{12}^{0}=0^{\circ}$ (no solar mixing) is also considered. Incorporating the corrections provided by the subdominant type-I seesaw involves degenerate perturbation theory due to vanishing solar splitting in the type-II seesaw enabling the solar mixing angle to receive substantial corrections. Apart from amending the solar sector, the type-I seesaw also tunes all the neutrino oscillation parameters into the allowed ranges, thus interrelating them all. Thus, the model is testable in the light of future experimental data. As an example, $\theta_{23}$ emerges in the first (second) octant for normal (inverted) ordering. $C P$-violation is controlled by phases present in the right-handed Majorana neutrino mass matrix, $M_{\nu R}$. Only normal ordering is allowed if these phases are absent. If $M_{\nu R}$ is complex the Dirac $C P$-violating phase $\delta$, can be large, i.e., $\sim \pm \pi / 2$, and inverted ordering is also allowed. T2K and NOVA preliminary data favoring normal ordering and $\delta \sim-\pi / 2$ predicts lightest neutrino mass to be $0.05 \mathrm{eV}$ or more within the model framework.
\end{abstract}

DOI: 10.1103/PhysRevD.98.075016

\section{INTRODUCTION}

Intensive experimental investigations worldwide have determined neutrino masses and mixing to a great extent. In spite of these, neutrinos retain certain mysteries including the ordering of their masses, their absolute mass scale, their Dirac or Majorana nature, the octant of the atmospheric mixing angle $\theta_{23}$ and $C P$-violation in lepton sector. While future experiments address these riddles, here a model of neutrino masses and mixing in concord with the experimental observations is proposed. The two small quantities $\theta_{13}$ and the ratio $R \equiv \Delta m_{\text {solar }}^{2} / \Delta m_{\text {atmos }}^{2}$ can get interrelated when both are

\footnotetext{
*soumita509@gmail.com
}

Published by the American Physical Society under the terms of the Creative Commons Attribution 4.0 International license. Further distribution of this work must maintain attribution to the author(s) and the published article's title, journal citation, and DOI. Funded by SCOAP . derived from a single perturbation [1]. In [2], larger mixing parameters like $\Delta m_{\text {atmos }}^{2}$ and $\theta_{23}=\pi / 4$ were ascribed to the dominant fundamental structure of neutrino masses and mixing, whereas the other oscillation parameters i.e., $\theta_{13}, \theta_{12}$, the deviation of $\theta_{23}$ from $\pi / 4$, and $\Delta m_{\text {solar }}^{2}$ originated from a smaller seesaw [3] generated perturbation. ${ }^{1}$ This induces constraints on the measured parameters. Certain symmetries can give rise to vanishing $\theta_{13}$ rather easily, and new models based on perturbations of such structures are also common in literature $[5,6]$.

Here, a schematic outline of the current exercise is given. The following standard parametrization of the lepton mixing matrix - the Pontecorvo, Maki, Nakagawa, Sakata (PMNS) matrix $-U$ has been used as

\footnotetext{
${ }^{1}$ Earlier attempts on neutrino mass models with some oscillation parameters much smaller than the others can be located in [4].
} 


$$
U=\left(\begin{array}{ccc}
c_{12} c_{13} & s_{12} c_{13} & s_{13} e^{-i \delta} \\
-c_{23} s_{12}+s_{23} s_{13} c_{12} e^{i \delta} & c_{23} c_{12}+s_{23} s_{13} s_{12} e^{i \delta} & s_{23} c_{13} \\
s_{23} s_{12}+c_{23} s_{13} c_{12} e^{i \delta} & -s_{23} c_{12}+c_{23} s_{13} s_{12} e^{i \delta} & c_{23} c_{13}
\end{array}\right),
$$

where $c_{i j}=\cos \theta_{i j}$ and $s_{i j}=\sin \theta_{i j}$. Neutrino masses and mixing are generated by a two-component Lagrangian, one of the dominant type-II seesaw structures, while the subdominant contribution originates from the type-I seesaw. The larger atmospheric mass splitting, $\Delta m_{\mathrm{atmos}}^{2}$, and maximal atomspheric mixing $\left(\theta_{23}=\pi / 4\right)$ are embedded within the type-II seesaw structure, whereas the solar splitting, $\Delta m_{\text {solar }}^{2}$ and $\theta_{13}$, are kept at zero. The solar mixing angle can vary continuously and acquire any desired value of $\theta_{12}^{0}$. Needless to mention, neither $\Delta m_{\text {solar }}^{2}$ nor $\theta_{13}$ are vanishing [7]. Evidences of nonmaximal yet large $\theta_{23}$ exist. The solar mixing angle $\theta_{12}$ is also constrained by experiments. The type-I seesaw alleviates all these issues. Since the solar splitting is vanishing in the type-II seesaw scenario, the first two mass eigenstates are degenerate. In order to lift this degeneracy with the help of the type-I seesaw contribution, one has to use degenerate perturbation theory. As a consequence of this, corrections to the solar mixing angle can be large.

The starting structure can be of tribimaximal (TBM), bimaximal (BM), and golden ratio (GR) mixings. All of these have $\theta_{13}=0$ and $\theta_{23}=\pi / 4$, with $\theta_{12}^{0}$ being the only discriminating factor as specified in Table I. In this Table, the fourth option corresponds to no solar mixing (NSM) i.e., $\theta_{12}^{0}=0$, which has the virtue of the mixing angles to be either maximal, i.e., $\pi / 4\left(\theta_{23}\right)$ or vanishing $\left(\theta_{13}\right.$ and $\left.\theta_{12}^{0}\right)$. An A4-based model with identical objectives only for the NSM case was studied in [8]. This attempt along with [8] differs from the other earlier works on $A 4$ [9-11], as in most of them, the neutrino mass matrix was derived as an outcome of a type-II seesaw mechanism, and obtaining TBM was of chief importance. Recent activities directed towards more realistic mixing patterns [12] often leading to breaking of $A 4$ symmetry can be found in [13].

A few distinctive aspects of this model are worth noting at this point. First, a combination of the type-I and type-II seesaw is considered. Second, the model is constructed to accommodate many popular mixing patterns. This is the first attempt of this kind using $A 4$ flavor symmetry that amends several popular lepton mixing patterns in a single stroke in which the type-II seesaw is the dominant

TABLE I. $\quad \theta_{12}^{0}$ for different popular lepton mixing patterns viz. TBM, BM, and GR mixing. NSM represents the case with vanishing solar mixing.

\begin{tabular}{lcccc}
\hline \hline Model & TBM & BM & GR & NSM \\
\hline$\theta_{12}^{0}$ & $35.3^{\circ}$ & $45.0^{\circ}$ & $31.7^{\circ}$ & $0.0^{\circ}$ \\
\hline \hline
\end{tabular}

contribution and the type-I seesaw is subdominant. The symmetries are broken spontaneously. Further, soft symmetry breaking terms are prohibited. All symmetry conserving terms are included in the Lagrangian. Scalars and leptons involved in the model are assigned suitable $A 4$ charges to implement this feature. An analogous pursuit based on $S 3 \times Z 3$ resulted in [14].

All the three neutrino mixing angles and the solar mass splitting receives first-order corrections from a single source - the type-I seesaw-in this model. Owing to the common origin, they all get interrelated. These correlations are characteristic features of this particular model. Indeed the model has a large number of parameters, but it must noted that only the region of the parameter space allowed by the neutrino mass and mixing data obeying these correlations is considered.

An analysis of the model initiates the discussion. In the next section, the operational strategy is described. The results so obtained are compared to the experimental data in the following section, succeeded by the conclusions and inferences of this work. Some essential ideas of the of the discrete symmetry $A 4$ are presented in Appendix A. A detailed study of the rich scalar sector to the extent of local minimization of the scalar potential is furnished in Appendix B. In Appendix C, algebraic details of the mass matrix calculations while going to the flavor basis of the neutrinos from the Lagrangian basis can be found.

\section{THE MASS MODEL}

The model comprises of scalars and leptons with specific A4 charges. All terms allowed by the symmetries under consideration are included in the Lagrangian. No soft symmetry-breaking term is included.

The right-handed charged leptons transform as $1\left(e_{R}\right)$, $1^{\prime}\left(\mu_{R}\right)$, and $1^{\prime \prime}\left(\tau_{R}\right)$ under $A 4$. The left-handed lepton doublets of three flavors constitute an $A 4$ triplet, so does

TABLE II. The lepton catalog of the model. The $A 4$ quantum numbers assignments of the fields are featured together with their $S U(2)_{L}$ properties. The hypercharge, $Y$, and lepton number, $L$, are displayed.

\begin{tabular}{lcccc}
\hline \hline Fields & Notations & $A 4$ & $S U(2)_{L}(Y)$ & $L$ \\
\hline Left-handed leptons & $\left(\nu_{i}, l_{i}\right)_{L}$ & 3 & $2(-1)$ & 1 \\
Right-handed charged leptons & $l_{1 R}$ & 1 & $1(-2)$ & 1 \\
& $l_{2 R}$ & $1^{\prime}$ & & \\
Right-handed neutrinos & $l_{3 R}$ & $1^{\prime \prime}$ & & \\
\hline \hline
\end{tabular}


the right-handed neutrinos. ${ }^{2}$ Table II shows the lepton constituents of the model together with their transformation properties under $A 4$ and $S U(2)_{L}$. The hypercharge and lepton number assignments are also shown. ${ }^{3}$ The choices of $A 4$ properties of the fields are not unique. A list of all possible options can be found in [15] of which this model adopts class B. The model is restricted to leptons only. ${ }^{4}$

Masses of all leptons originate from A4-invariant Yukawa couplings. Several scalar fields have to be included $^{5}$ that acquire suitable vacuum expectation values (vevs). The strategy of choosing the scalar field multiplets requires some elaboration. An idea of the mass matrices of the left- and right-handed neutrinos in the flavor basis (charged lepton mass matrix diagonal) that are suitable for our avowed goal can be acquired from our previous work [14]. The Lagrangian is written down in a basis which is unitarily related to the flavor basis. Consequently, the mass matrices in this defining basis have somewhat complicated structures for which the motivation is not initially obvious. These forms of the mass matrices (below) arise from a rather large set of scalars and their vevs.

The charged leptons acquire their masses through the $S U(2)_{L}$ doublet scalar fields $\Phi_{i}(i=1,2,3)$ forming an $A 4$ triplet. The neutrino Dirac mass matrix is generated by an $A 4$ invariant $S U(2)_{L}$ doublet $\eta$, having lepton number 2 .
$S U(2)_{L}$ triplet scalars are required for the type-II seesaw for left-handed neutrino mass matrix that include $A 4$ triplet fields $\hat{\Delta}_{a}^{L}$ and $\hat{\Delta}_{b}^{L}$ along with $\Delta_{\zeta}^{L}, \zeta=1,2,3$ transforming as $1,1^{\prime}, 1^{\prime \prime}$ of $A 4$. These are used to construct the dominant type-II seesaw neutrino mass matrix. Effects of the subdominant type-I seesaw contribution is included perturbatively. A4 conserving Yukawa couplings produce the right-handed neutrino mass matrix as well. Several $S U(2)_{L}$ singlet scalars are involved in generation of the Majorana masses for the right-handed neutrinos viz. $\hat{\Delta}_{p}^{R}$ $(p=a, b, c)$ transforming as $A 4$ triplets and $\Delta_{\gamma}^{R}(\gamma=1,2$, 3 ) transforming as $1,1^{\prime}$ and $1^{\prime \prime}$ under $A 4$. Table III evinces transformation properties of the model scalars under $A 4$ and $S U(2)_{L}$ together with their hypercharge, lepton number and vev configurations. The vevs of the $S U(2)_{L}$ doublet scalars are of $\mathcal{O}\left(M_{W}\right)$ while that of the $S U(2)_{L}$ triplets are several orders of magnitude smaller than the doublet vevs in concord with the small neutrino masses as well as the $\rho$ parameter of electroweak symmetry breaking. As expected, the vevs of the $S U(2)_{L}$ singlets responsible for righthanded neutrino mass lies much above the electroweak scale. The mass terms of the neutrinos (both type-I and type-II seesaw) and that of the charged leptons are generated by a $S U(2)_{L} \times U(1)_{Y}$ conserving Lagrangian that preserves $A 4$ as well ${ }^{6}$ :

$$
\begin{aligned}
\mathcal{L}_{\text {mass }}= & y_{j} \rho_{j i k} \bar{l}_{L i} l_{R j} \Phi_{k}^{0} \quad \text { (charged lepton mass) }+f \rho_{1 i k} \bar{\nu}_{L i} N_{R k} \eta^{0} \quad \text { (neutrino Dirac mass) } \\
& +\frac{1}{2}\left(\sum_{n=a, b} \hat{Y}_{n}^{L} \alpha_{i j k} \nu_{L i}^{T} C^{-1} \nu_{L j} \hat{\Delta}_{n k}^{L 0}+Y_{\zeta}^{L} \rho_{\zeta i j} \nu_{L i}^{T} C^{-1} \nu_{L j} \Delta_{\zeta}^{L 0}\right) \quad \text { (neutrino type-II seesaw mass) } \\
& +\frac{1}{2}\left(\sum_{p=a, b, c} \hat{Y}_{p}^{R} \alpha_{i j k} N_{R i}^{T} C^{-1} N_{R j} \hat{\Delta}_{k p}^{R 0}+Y_{\gamma}^{R} \rho_{\gamma i j} N_{R i}^{T} C^{-1} N_{R j} \Delta_{\gamma}^{R 0}\right) \quad \text { (rh neutrino mass) + H.c. }
\end{aligned}
$$

The scalars acquire the following vevs $\left(S U(2)_{L}\right.$ part is suppressed):

$$
\begin{gathered}
\left\langle\Phi^{0}\right\rangle=\frac{v}{\sqrt{3}}\left(\begin{array}{l}
1 \\
1 \\
1
\end{array}\right), \quad\left\langle\eta^{0}\right\rangle=u, \quad\left\langle\hat{\Delta}_{a}^{L 0}\right\rangle=v_{L a}\left(\begin{array}{l}
1 \\
0 \\
0
\end{array}\right), \quad\left\langle\hat{\Delta}_{b}^{L 0}\right\rangle=v_{L b}\left(\begin{array}{l}
1 \\
1 \\
1
\end{array}\right), \quad\left\langle\Delta_{1}^{L 0}\right\rangle=\left\langle\Delta_{2}^{L 0}\right\rangle=\left\langle\Delta_{3}^{L 0}\right\rangle=u_{L}, \\
\left\langle\hat{\Delta}_{a}^{R 0}\right\rangle=v_{R a}\left(\begin{array}{l}
1 \\
1 \\
1
\end{array}\right), \quad\left\langle\hat{\Delta}_{b}^{R 0}\right\rangle=v_{R b}\left(\begin{array}{c}
1 \\
\omega \\
\omega^{2}
\end{array}\right), \quad\left\langle\hat{\Delta}_{c}^{R 0}\right\rangle=v_{R c}\left(\begin{array}{c}
1 \\
\omega^{2} \\
\omega
\end{array}\right),
\end{gathered}
$$

\footnotetext{
${ }^{2}$ The notation followed closely resembles that of [9].

${ }^{3}$ Opposite lepton numbers are assigned to $\nu_{L}$ and $N_{R}$ in order to prohibit their coupling with $\Phi$ so that the Dirac mass matrix can remain proportional to the identity matrix.

${ }_{5}^{4}$ Quark models based on $A 4$ has been explored in [16,17].

${ }^{5}$ Models addressing this issue by separating the breaking of $S U(2)_{L}$ and $A 4$ are widely studied in literature [10]. The former is mediated by the usual doublet and triplet scalars of $S U(2)_{L}$ that are invariant under $A 4$. The breaking of $A 4$ is induced by the vev of "flavon" scalar fields that are singlets of $S U(2)_{L}$ but their transformations under $A 4$ is nontrivial. Though such models are economic effective dimension-5 interactions comes into play in order to connect the fermions with the two types of scalar fields simultaneously leading to an interpretation as an effective theory.

${ }^{6}$ Lepton number is also conserved for the mass terms of Dirac kind.
} 
TABLE III. The scalar sector of the model. The $A 4$ charges as well as the $S U(2)_{L}$ nature of the scalars are exhibited. The hypercharge, $Y$, lepton number, $L$, and the vacuum expectation value (vev) configurations of the scalars are also presented.

\begin{tabular}{|c|c|c|c|c|c|}
\hline Purpose & Notations & $A 4$ & $S U(2)_{L}(Y)$ & $L$ & Vev \\
\hline Charged fermion mass & $\Phi=\left(\begin{array}{ll}\phi_{1}^{+} & \phi_{1}^{0} \\
\phi_{2}^{+} & \phi_{2}^{0} \\
\phi_{3}^{+} & \phi_{3}^{0}\end{array}\right)$ & 3 & $2(1)$ & 0 & $\langle\Phi\rangle=\frac{v}{\sqrt{3}}\left(\begin{array}{ll}0 & 1 \\
0 & 1 \\
0 & 1\end{array}\right)$ \\
\hline Neutrino Dirac mass & $\eta=\left(\eta^{0}, \eta^{-}\right)$ & 1 & $2(-1)$ & 2 & $\langle\eta\rangle=(u, 0)$ \\
\hline Type-II seesaw mass & $\hat{\Delta}_{a}^{L}=\left(\begin{array}{ccc}\hat{\Delta}_{1 a}^{++} & \hat{\Delta}_{1 a}^{+} & \hat{\Delta}_{1 a}^{0} \\
\hat{\Delta}_{2 a}^{++} & \hat{\Delta}_{2 a}^{+} & \hat{\Delta}_{2 a}^{0} \\
\hat{\Delta}_{3 a}^{++} & \hat{\Delta}_{3 a}^{+} & \hat{\Delta}_{3 a}^{0}\end{array}\right)^{L}$ & 3 & $3(2)$ & -2 & $\left\langle\hat{\Delta}_{a}^{L}\right\rangle=v_{L a}\left(\begin{array}{ccc}0 & 0 & 1 \\
0 & 0 & 1 \\
0 & 0 & 1\end{array}\right)$ \\
\hline Type-II seesaw mass & $\hat{\Delta}_{b}^{L}=\left(\begin{array}{ccc}\hat{\Delta}_{1 b}^{++} & \hat{\Delta}_{1 b}^{+} & \hat{\Delta}_{1 b}^{0} \\
\hat{\Delta}_{2 b}^{++} & \hat{\Delta}_{2 b}^{+} & \hat{\Delta}_{2 b}^{0} \\
\hat{\Delta}_{3 b}^{++} & \hat{\Delta}_{3 b}^{+} & \hat{\Delta}_{3 b}^{0}\end{array}\right)^{L}$ & 3 & $3(2)$ & -2 & $\left\langle\hat{\Delta}_{b}^{L}\right\rangle=v_{L b}\left(\begin{array}{ccc}0 & 0 & 1 \\
0 & 0 & 1 \\
0 & 0 & 1\end{array}\right)$ \\
\hline & & 1 & $3(2)$ & -2 & $\left\langle\Delta_{1}^{L}\right\rangle=\left(0,0, u_{L}\right)$ \\
\hline Type-II seesaw mass & $\Delta_{\zeta}^{L}=\left(\Delta_{\zeta}^{++}, \Delta_{\zeta}^{+}, \Delta_{\zeta}^{0}\right)^{L}$ & $\begin{array}{l}1^{\prime} \\
1^{\prime \prime}\end{array}$ & $\begin{array}{l}3(2) \\
3(2)\end{array}$ & $\begin{array}{l}-2 \\
-2\end{array}$ & $\begin{array}{l}\left\langle\Delta_{2}^{L}\right\rangle=\left(0,0, u_{L}\right) \\
\left\langle\Delta_{3}^{L}\right\rangle=\left(0,0, u_{L}\right)\end{array}$ \\
\hline Right-handed neutrino mass & $\hat{\Delta}_{a}^{R}=\left(\begin{array}{c}\hat{\Delta}_{1 a}^{0} \\
\hat{\Delta}_{2 a}^{0} \\
\hat{\Delta}_{3 a}^{0}\end{array}\right)^{R}$ & 3 & $1(0)$ & 2 & $\left\langle\hat{\Delta}_{a}^{R}\right\rangle=v_{R a}\left(\begin{array}{l}1 \\
1 \\
1\end{array}\right)$ \\
\hline Right-handed neutrino mass & $\hat{\Delta}_{b}^{R}=\left(\begin{array}{c}\hat{\Delta}_{1 b}^{0} \\
\hat{\Delta}_{2 b}^{0} \\
\hat{\Delta}_{3 b}^{0}\end{array}\right)^{R}$ & 3 & $1(0)$ & 2 & $\left\langle\hat{\Delta}_{b}^{R}\right\rangle=v_{R b}\left(\begin{array}{c}1 \\
\omega \\
\omega^{2}\end{array}\right)$ \\
\hline Right-handed neutrino mass & $\hat{\Delta}_{c}^{R}=\left(\begin{array}{c}\hat{\Delta}_{1 c}^{0} \\
\hat{\Delta}_{2 c}^{0} \\
\hat{\Delta}_{3 c}^{0}\end{array}\right)^{R}$ & 3 & $1(0)$ & 2 & $\left\langle\hat{\Delta}_{c}^{R}\right\rangle=v_{R c}\left(\begin{array}{c}1 \\
\omega^{2} \\
\omega\end{array}\right)$ \\
\hline Right-handed neutrino mass & $\Delta_{1}^{R}=\left(\Delta_{1}^{0}\right)^{R}$ & 1 & $1(0)$ & 2 & $\left\langle\Delta_{1}^{R}\right\rangle=u_{1 R}$ \\
\hline Right-handed neutrino mass & $\Delta_{2}^{R}=\left(\Delta_{2}^{0}\right)^{R}$ & $1^{\prime}$ & $1(0)$ & 2 & $\left\langle\Delta_{2}^{R}\right\rangle=u_{2 R}$ \\
\hline Right-handed neutrino mass & $\Delta_{3}^{R}=\left(\Delta_{3}^{0}\right)^{R}$ & $1^{\prime \prime}$ & $1(0)$ & 2 & $\left\langle\Delta_{3}^{R}\right\rangle=u_{3 R}$ \\
\hline
\end{tabular}

$\left\langle\Delta_{1}^{R 0}\right\rangle=u_{1 R}, \quad\left\langle\Delta_{2}^{R 0}\right\rangle=u_{2 R}, \quad\left\langle\Delta_{3}^{R 0}\right\rangle=u_{3 R}$

An elaborate study of the $A 4$ conserving scalar potential involving the fields listed in Table III is presented in Appendix B of this paper. Local minimization is performed and the conditions corresponding to the particular vev structures as indicated in Eqs. (3)-(5) are obtained.

The mass matrix for the charged leptons and the lefthanded Majorana neutrinos so obtained are:

$$
\begin{aligned}
M_{e \mu \tau}= & \frac{v}{\sqrt{3}}\left(\begin{array}{ccc}
y_{1} & y_{2} & y_{3} \\
y_{1} & \omega y_{2} & \omega^{2} y_{3} \\
y_{1} & \omega^{2} y_{2} & \omega y_{3}
\end{array}\right), \\
M_{\nu L}= & \left(\begin{array}{ccc}
\left(Y_{1}^{L}+2 Y_{2}^{L}\right) u_{L} & \frac{1}{2} \hat{Y}_{b}^{L} v_{L b} & \frac{1}{2} \hat{Y}_{b}^{L} v_{L b} \\
\frac{1}{2} \hat{Y}_{b}^{L} v_{L b} & \left(Y_{1}^{L}-Y_{2}^{L}\right) u_{L} & \frac{1}{2}\left(\hat{Y}_{a}^{L} v_{L a}+\hat{Y}_{b}^{L} v_{L b}\right) \\
\frac{1}{2} \hat{Y}_{b}^{L} v_{L b} & \frac{1}{2}\left(\hat{Y}_{a}^{L} v_{L a}+\hat{Y}_{b}^{L} v_{L b}\right) & \left(Y_{1}^{L}-Y_{2}^{L}\right) u_{L}
\end{array}\right),
\end{aligned}
$$


where the choice of $Y_{2}^{L}=Y_{3}^{L}$ is made. The Yukawa couplings involved in the charged lepton mass matrix satisfies $y_{1} v=m_{e}, y_{2} v=m_{\mu}, y_{3} v=m_{\tau}$. The neutrino mass matrix of Dirac nature and the right-handed neutrino mass matrix of Majorana kind acquires the following structures:

$$
M_{D}=f u \mathbb{I}, \quad M_{\nu R}=m_{R}\left(\begin{array}{ccc}
\chi_{1} & \chi_{6} & \chi_{5} \\
\chi_{6} & \chi_{2} & \chi_{4} \\
\chi_{5} & \chi_{4} & \chi_{3}
\end{array}\right)
$$

$m_{D}$ sets the scale of Dirac masses of the neutrinos where one can identify $f u=m_{D}$. The scale of the type-II seesaw neutrino masses is much smaller than that of the charged leptons i.e., $\mathcal{O}\left(M_{\nu L}\right) \sim u_{L}, v_{L a}, v_{L b}$ where $u_{L}, v_{L a}, v_{L b} \ll v$. Such a possibility that the triplet vev is much smaller than the doublet vev can be obtained as shown in [18], albeit in a model with fewer scalars. The scale of the right-handed Majorana neutrino masses is set by $m_{R}$ and $\chi_{i}$ in Eq. (7) are dimensionless quantities ${ }^{7}$ of $\mathcal{O}(1)$.

The mass matrices in Eq. (6) could be expressed in a more convenient form by applying a couple of transformations. The non-Hermitian charged lepton mass matrix can be diagonalized by applying a transformation $U_{L}$ (below) on the left-handed lepton doublets and no transformation on the right-handed charged leptons. The transformation matrices are expressed as

$$
U_{L}=\frac{1}{\sqrt{3}}\left(\begin{array}{ccc}
1 & 1 & 1 \\
1 & \omega^{2} & \omega \\
1 & \omega & \omega^{2}
\end{array}\right)
$$

This basis in which the charged lepton mass matrix is diagonal and the entire lepton mixing is governed by the neutrino sector is termed as the flavor basis in which the mass matrices acquire the following forms:

$$
\begin{aligned}
M_{e \mu \tau}^{\text {flavor }} & =\left(\begin{array}{ccc}
m_{e} & 0 & 0 \\
0 & m_{\mu} & 0 \\
0 & 0 & m_{\tau}
\end{array}\right), \\
M_{\nu L}^{\text {flavor }} & =\frac{1}{2}\left(\begin{array}{ccc}
2 m_{1}^{(0)} & 0 & 0 \\
0 & m^{+} & m^{-} \\
0 & m^{-} & m^{+}
\end{array}\right) .
\end{aligned}
$$

Here $m^{ \pm} \equiv m_{3}^{(0)} \pm m_{1}^{(0)}$. Therefore, $m^{-}$is positive (negative) for normal (inverted) ordering. As noted earlier, $M_{\nu L}^{\text {flavor }}$, which arises from the type-II seesaw, is the dominant contribution to the neutrino mass.

\footnotetext{
${ }^{7}$ See Appendix C for exact expressions of $\chi_{i}$ in Eq. (7).
}

Demanding that the neutrino Dirac mass matrix, which couples the left- and right-handed neutrinos, preserves its proportionality to the identity matrix necessitates that the transformation applied on the right-handed neutrino fields must be $V_{R}=U_{L}$. Thus, we get

$$
\begin{aligned}
M_{D} & =f u \mathbb{I}, \\
M_{\nu R}^{\mathrm{flavor}} & =\left(V_{R}^{\dagger} M_{\nu R} V_{R}^{\dagger}\right)=\frac{m_{R}}{4 a b}\left(\begin{array}{lll}
r_{11} & r_{12} & r_{13} \\
r_{12} & r_{22} & r_{23} \\
r_{13} & r_{23} & r_{33}
\end{array}\right) .
\end{aligned}
$$

The matrices in Eq. (10) will take part in the type-I seesaw mechanism. ${ }^{8}$ Various identification of the products of the Yukawa couplings and the vevs with the neutrino mass and mixing parameters are necessary for the mass matrices to be expressed in the forms as presented in Eqs. (9) and (10). Appendix $\mathrm{C}$ comprises of these algebraic details.

\section{MODUS OPERANDI}

The four mass matrices in the flavor basis obtained from the model are given in Eqs. (9) and (10). In this basis the entire lepton mixing and $C P$-violation is controlled solely by the neutrino sector to which we restrict our discussion now onwards. The subdominant contribution given by the type-I seesaw is incorporated by perturbation theory to the dominant component $M_{\nu L}^{\text {flavor }}$ coming from the type-II seesaw. The flavor basis mass matrices have to undergo one more basis transformations for successful implementation of this scheme. More precisely they ought to be expressed in the mass basis of the neutrinos which by definition has the left-handed neutrino mass matrix diagonal in it. Thus,

$M^{0}=M_{\nu L}^{\text {mass }}=U^{0 T} M_{\nu L}^{\mathrm{flavor}} U^{0}=\left(\begin{array}{ccc}m_{1}^{(0)} & 0 & 0 \\ 0 & m_{1}^{(0)} & 0 \\ 0 & 0 & m_{3}^{(0)}\end{array}\right)$,

where

$$
U^{0}=\left(\begin{array}{ccc}
\cos \theta_{12}^{0} & \sin \theta_{12}^{0} & 0 \\
-\frac{\sin \theta_{12}^{0}}{\sqrt{2}} & \frac{\cos \theta_{12}^{0}}{\sqrt{2}} & \frac{1}{\sqrt{2}} \\
\frac{\sin \theta_{12}^{0}}{\sqrt{2}} & -\frac{\cos \theta_{12}^{0}}{\sqrt{2}} & \frac{1}{\sqrt{2}}
\end{array}\right) .
$$

The left-handed neutrino fields in the mass basis $\left(\left|\nu_{L}^{\text {mass }}\right\rangle\right)$ are connected to the ones in the flavor basis $\left(\left|\nu_{L}^{\text {flavor }}\right\rangle\right)$ by this $U^{0}$ furnished in Eq. (12). One can obtain the $\left|\nu_{L}^{\text {mass }}\right\rangle$ by applying $U^{0 \dagger}$ on $\left|\nu_{L}^{\text {flavor }}\right\rangle$ i.e., $\left|\nu_{L}^{\text {mass }}\right\rangle=U^{0 \dagger}\left|\nu_{L}^{\text {flavor }}\right\rangle$. It immediately follows from Eqs. (11), (1) and (12) that, in the type-II seesaw component, solar splitting is absent,

\footnotetext{
${ }^{8}$ Explicit forms of $r_{i j}$ in Eq. (10) can be found in Appendix C.
} 
$\theta_{13}=0$ and $\theta_{23}=\pi / 4$. The columns of $U^{0}$ are the unperturbed flavor basis.

Once again we demand that in the mass basis the neutrino Dirac mass matrix remains proportional to identity. In order to satisfy this, the same transformation $\left(U^{0 \dagger}\right)$ has to be applied on the right-handed neutrino fields. This leads to changes in form of right-handed neutrino mass matrix given by $M_{\nu R}^{\text {mass }}=\left(U^{0 \dagger} M_{\nu R}^{\text {flavor }} U^{0}\right)$. The matrices contributing in the type-I seesaw are as follows:

$$
M_{D}=m_{D} \mathbb{I} \quad \text { and } \quad M_{\nu R}^{\text {mass }}=\frac{m_{R}}{2 \sqrt{2} a b}\left(\begin{array}{ccc}
0 & b & b \\
b & \frac{a}{\sqrt{2}} & -\frac{a}{\sqrt{2}} \\
b & -\frac{a}{\sqrt{2}} & \frac{a}{\sqrt{2}}
\end{array}\right) \text {. }
$$

Here $a$ and $b$ are dimensionless quantities ${ }^{9}$ of $\mathcal{O}(1)$. It is imperative to note that $a$ and $b$ can in general be complex. One can in principle trade off $a$ and $b$ in terms of complex numbers $y e^{-i \phi_{2}}$ and $x e^{-i \phi_{1}}$ respectively, where $x$ and $y$ are dimensionless real quantities of $\mathcal{O}(1)$. The type-I seesaw contribution is obtained by

$$
M^{\prime}=\left[M_{D}^{T}\left(M_{\nu R}\right)^{-1} M_{D}\right]=\frac{m_{D}^{2}}{m_{R}}\left(\begin{array}{ccc}
0 & y e^{i \phi_{1}} & y e^{i \phi_{1}} \\
y e^{i \phi_{1}} & \frac{x e^{i \phi_{2}}}{\sqrt{2}} & \frac{-x e^{i \phi_{2}}}{\sqrt{2}} \\
y e^{i \phi_{1}} & \frac{-x e^{i \phi_{2}}}{\sqrt{2}} & \frac{x e^{i \phi_{2}}}{\sqrt{2}}
\end{array}\right) .
$$

Here the Dirac mass matrix is proportional to identity. It was checked that the same results can follow as long as $M_{D}$ is diagonal. $M_{\nu R}^{\text {mass }}$ exhibits a $N_{2 R} \leftrightarrow N_{3 R}$ discrete symmetry. The results remain intact even if that choice is relaxed. Now onwards the entire procedure is carried on in the mass basis of the neutrinos using the mass matrices expressed in Eqs. (11) and (14).

The method followed below essentially consists of the following steps. From the type-II seesaw, a lepton mixing of the form of Eq. (12) is generated, with $\theta_{12}^{0}$ of any preferred value. At this stage, only the atmospheric mass splitting is nonzero and atmospheric mixing is maximal. Next, the type-I seesaw is included using degenerate perturbation theory. The solar mass splitting and the desired $\theta_{12}$ are first obtained. Then the third column of the mixing matrix is calculated and compared with Eq. (1) to extract $\theta_{13}, \theta_{23}$, and $\delta$.

\section{RESULTS}

The neutrino mass matrices derived from the type-I and type-II seesaw mechanism have been discussed in the previous section, of which the former is significantly smaller than the latter. In the absence of the type-I seesaw contribution, the leptonic mixing matrix characterized by

\footnotetext{
${ }^{9}$ See Eq. (C5) in Appendix C for details.
}

$\theta_{13}=0, \theta_{23}=\pi / 4$, and $\theta_{12}^{0}$ is free to vary. Consequences for the four choices of the value of $\theta_{12}^{0}$, corresponding to the TBM, BM, GR, and NSM cases together with the vanishing solar splitting, are examined. This, along with the atmospheric mass splitting allowed by the data, depict the type-II seesaw structure. Inclusion of type-I seesaw corrections perturbatively up to first order modulates the neutrino oscillation parameters into the ranges preferred by data. Owing to the vanishing solar splitting in the type-II seesaw contribution, the first two mass eigenstates are degenerate. Thus, in the solar sector degenerate perturbation theory has to be applied. Hence, the first-order corrections to the solar mixing angle can be large. The global best-fit of the oscillation parameters are displayed in the next section.

\section{A. Data}

The current $3 \sigma$ global fits of the neutrino oscillation parameters are: $[19,20]$

$$
\begin{aligned}
\Delta m_{21}^{2} & =(7.02-8.08) \times 10^{-5} \mathrm{eV}^{2}, \\
\theta_{12} & =(31.52-36.18)^{\circ}, \\
\left|\Delta m_{31}^{2}\right| & =(2.351-2.618) \times 10^{-3} \mathrm{eV}^{2}, \\
\theta_{23} & =(38.6-53.1)^{\circ}, \\
\theta_{13} & =(7.86-9.11)^{\circ}, \quad \delta=(0-360)^{\circ} .
\end{aligned}
$$

These numbers are taken from NuFIT2.1 of 2016 [19]. Needless to mention, $\Delta m_{i j}^{2} \equiv m_{i}^{2}-m_{j}^{2}$, such that $\Delta m_{31}^{2}>0$ for normal ordering (NO) and $\Delta m_{31}^{2}<0$ for inverted ordering (IO). Two best-fit points of $\theta_{23}$ are evinced by the data in the first and in the second octants. Towards the end of the paper it is discussed how the model can accommodate the recent T2K and NOVA hints [21,22] of $\delta$ close to $-\pi / 2$.

\section{B. Real $M_{\nu R}\left(\phi_{1}=0\right.$ or $\pi, \phi_{2}=0$ or $\left.\pi\right)$}

As a warm-up exercise let us consider the simpler case of $M_{\nu R}$ real. In such a scenario there is no $C P$-violation as the phases $\phi_{1,2}$ of Eq. (14) are 0 or $\pi$. This leads to four different alternatives available for choosing $\phi_{1}$ and $\phi_{2}$. These are captured compactly by taking $x$ and $y$ real and allowing them to assume both signs for notational convenience. It will be soon clear how the experimental observations prefer one or the other of these four alternatives. Thus, for real $M_{\nu R}$, the type-I seesaw contribution appears like:

$$
M^{\prime}=\frac{m_{D}^{2}}{m_{R}}\left(\begin{array}{ccc}
0 & y & y \\
y & \frac{x}{\sqrt{2}} & -\frac{x}{\sqrt{2}} \\
y & -\frac{x}{\sqrt{2}} & \frac{x}{\sqrt{2}}
\end{array}\right) .
$$

The degeneracy of the two neutrino masses in the type-II seesaw ensuring the vanishing solar splitting necessitates 
TABLE IV. Data allowed $3 \sigma$ ranges of $\zeta$ [Eq. (18)], $\epsilon$ [Eq. (19)], and $\left(\epsilon-\theta_{12}^{0}\right)$ for different popular mixing patterns are shown.

\begin{tabular}{lcccc}
\hline \hline Model $\left(\theta_{12}^{0}\right)$ & TBM $\left(35.3^{\circ}\right)$ & BM $\left(45.0^{\circ}\right)$ & GR $\left(31.7^{\circ}\right)$ & NSM $\left(0.0^{\circ}\right)$ \\
\hline$\zeta$ & $-4.0^{\circ} \leftrightarrow 0.6^{\circ}$ & $-13.7^{\circ} \leftrightarrow-9.1^{\circ}$ & $-0.4^{\circ} \leftrightarrow 4.2^{\circ}$ & $31.3^{\circ} \leftrightarrow 35.9^{\circ}$ \\
$\epsilon$ & $-4.0^{\circ} \leftrightarrow 0.6^{\circ}$ & $-14.5^{\circ} \leftrightarrow-9.3^{\circ}$ & $-0.4^{\circ} \leftrightarrow 4.2^{\circ}$ & $44.0^{\circ} \leftrightarrow 56.7^{\circ}$ \\
$\epsilon-\theta_{12}^{0}$ & $-39.2^{\circ} \leftrightarrow-34.6^{\circ}$ & $-59.5^{\circ} \leftrightarrow-54.4^{\circ}$ & $-39.2^{\circ} \leftrightarrow-30.0^{\circ}$ & $44.0^{\circ} \leftrightarrow 56.7^{\circ}$ \\
\hline \hline
\end{tabular}

the application of degenerate perturbation theory to obtain the corrections for the solar sector mixing parameters. ${ }^{10}$ The entire dynamics of this sector is dictated by the upper $2 \times 2$ submatrix of $M^{\prime}$ given by:

$$
M_{2 \times 2}^{\prime}=\frac{m_{D}^{2}}{m_{R}}\left(\begin{array}{cc}
0 & y \\
y & x / \sqrt{2}
\end{array}\right) .
$$

This gives rise to

$$
\theta_{12}=\theta_{12}^{0}+\zeta, \quad \tan 2 \zeta=2 \sqrt{2}\left(\frac{y}{x}\right)
$$

For functional ease, it is useful to define a quantity $\epsilon$ as

$$
\sin \epsilon=\frac{y}{\sqrt{y^{2}+x^{2} / 2}} \quad \text { and } \quad \cos \epsilon=\frac{x / \sqrt{2}}{\sqrt{y^{2}+x^{2} / 2}},
$$

i.e., $\quad \tan \epsilon=\frac{1}{2} \tan 2 \zeta$.

Once a mixing pattern is selected, the corresponding $\theta_{12}^{0}$ gets fixed and the experimental bounds of $\theta_{12}$ determines the $3 \sigma$ ranges of $\zeta$ and $\epsilon$ by means of Eqs. (15) and (19) as featured in Table IV. The ratio $(y / x)$ is positive (negative) when $\zeta$ is positive (negative). From Eq. (19), it is evident that the sign of $y$ is regulated by the value of $\epsilon$. Putting all these facts together it is easy to infer that $x$ is positive always, or in other words $\phi_{2}$ must be 0 , while $y$ has to be positive, $\phi_{1}=0$ (negative, $\phi_{1}=\pi$ ) for NSM (BM). In case of TBM and GR, both signs of $y$ are admissible. The solar splitting provided by the type-I seesaw as extracted from Eq. (17) is

$\Delta m_{\mathrm{solar}}^{2}=\frac{\sqrt{2} m_{D}^{2}}{m_{R}} m_{1}^{(0)} \sqrt{x^{2}+8 y^{2}}=\frac{\sqrt{2} m_{D}^{2}}{m_{R}} m_{1}^{(0)} \frac{x}{\cos 2 \zeta}$.

For the mass basis form of the mass matrix in Eq. (11), the mixing in the leptonic sector is completely given by the $U^{0}$ given in Eq. (12). After including the type-I seesaw

\footnotetext{
${ }^{10}$ Since degenerate perturbation theory is used in the solar sector, the first-order correction to the solar mixing angle $\zeta$ is not constrained to be small.
}

correction to the mass matrices, there is a further contribution to the mixing matrix as well, now given by

$\begin{aligned} U & =U^{0} U_{\nu} \\ \text { where } U_{\nu} & =\left(\begin{array}{ccc}\cos \zeta & -\sin \zeta & \kappa_{r} \sin \epsilon \\ \sin \zeta & \cos \zeta & -\kappa_{r} \cos \epsilon \\ \kappa_{r} \sin (\zeta-\epsilon) & \kappa_{r} \cos (\zeta-\epsilon) & 1\end{array}\right)\end{aligned}$

with

$$
\kappa_{r} \equiv \frac{m_{D}^{2}}{m_{R} m^{-}} \sqrt{y^{2}+x^{2} / 2}=\frac{m_{D}^{2}}{m_{R} m^{-}} \frac{x}{\sqrt{2} \cos \epsilon} .
$$

The third column of the lepton mixing matrix is:

$$
\left|\psi_{3}\right\rangle=\left(\begin{array}{c}
\kappa_{r} \sin \left(\epsilon-\theta_{12}^{0}\right) \\
\frac{1}{\sqrt{2}}\left[1-\kappa_{r} \cos \left(\epsilon-\theta_{12}^{0}\right)\right] \\
\frac{1}{\sqrt{2}}\left[1+\kappa_{r} \cos \left(\epsilon-\theta_{12}^{0}\right)\right]
\end{array}\right)
$$

As already pointed out, $x$ is always positive, $\kappa_{r}$ is positive (negative) for NO (IO).

Eq. (23) when mapped to the third column of Eq. (1) leads to

$$
\sin \theta_{13} \cos \delta=\kappa_{r} \sin \left(\epsilon-\theta_{12}^{0}\right)
$$

and

$$
\tan \left(\pi / 4-\theta_{23}\right) \equiv \tan \omega=\kappa_{r} \cos \left(\epsilon-\theta_{12}^{0}\right)
$$

The allowed ranges of $\left(\epsilon-\theta_{12}^{0}\right)$ for the different mixing patterns is given in Table IV. The $C P$-phase $\delta$ is $0(\pi)$ when $\sin \left(\epsilon-\theta_{12}^{0}\right)$ is positive (negative) in case of normal ordering. ${ }^{11}$ It can be immediately concluded that $\delta=0$ for the NSM from Table IV and $\delta=\pi$ for the rest of the options under study. $C P$ is conserved for both the values of $\delta$.

Using Eqs. (20), (22), and (24), we find

$$
\Delta m_{\mathrm{solar}}^{2}=2 m^{-} m_{1}^{(0)} \frac{\sin \theta_{13} \cos \delta \cos \epsilon}{\cos 2 \zeta \sin \left(\epsilon-\theta_{12}^{0}\right)} .
$$

\footnotetext{
${ }^{11}$ Inverted ordering is prohibited for real $M_{\nu R}$.
} 
For real $M_{\nu R}$ inverted ordering is forbidden as can be seen from Eq. (26). In order to justify this, one can define

$$
z \equiv m^{-} m_{1}^{(0)} / \Delta m_{\mathrm{atmos}}^{2} \quad \text { and } \quad \tan \xi \equiv m_{0} / \sqrt{\left|\Delta m_{\mathrm{atmos}}^{2}\right|}
$$

where $z$ is positive for both the orderings of neutrino masses. With the help of Eq. (26) it can be written as

$$
z=\left(\frac{\Delta m_{\text {solar }}^{2}}{\left|\Delta m_{\text {atmos }}^{2}\right|}\right)\left(\frac{\cos 2 \zeta \sin \left(\epsilon-\theta_{12}^{0}\right)}{2 \sin \theta_{13}|\cos \delta| \cos \epsilon}\right) .
$$

From Eq. (27), it is straightforward to show that

$$
\begin{aligned}
& z=\sin \xi /(1+\sin \xi) \text { i.e., } 0 \leq z \leq \frac{1}{2} \text { (fornormalordering), } \\
& z=1 /(1+\sin \xi) \text { i.e., } \frac{1}{2} \leq z \leq 1 \quad \text { (forinverted ordering). }
\end{aligned}
$$

The lightest neutrino mass $m_{0}$ has a one-to-one correspondence with $z$. In the quasidegenerate limit, i.e., $m_{0} \rightarrow$ large, $z \rightarrow \frac{1}{2}$ for both orderings. For real $M_{\nu R},|\cos \delta|=1$ in Eq. (28). It simply follows from the global fit mass splittings and mixing angles in Sec. IV A and Table IV that $z \sim 10^{-2}$ or smaller for all four popular mixing alternatives. Thus, inverted ordering is forbidden for real $M_{\nu R}$.

Using Eqs. (24) and (25), the deviation of the atmospheric mixing angle from maximality is found to be:

$$
\tan \omega=\frac{\sin \theta_{13} \cos \delta}{\tan \left(\epsilon-\theta_{12}^{0}\right)} .
$$

Eq. (25) implies that $\omega$ is positive always for normal ordering irrespective of the mixing pattern. Thus, $\theta_{23}$ is confined only to the first octant for real $M_{\nu R} . \epsilon$ can be expressed in terms of $\theta_{12}$ using Eqs. (18) and (19). Thus, $\omega$ in Eq. (30) can be expressed as a function of $\theta_{13}$ and $\theta_{12}$ only. Figure 1 exhibits $\omega$ as a function of $\theta_{12}$ for BM (thin pink lines) and NSM (thick green lines) alternatives. $\theta_{12}$ and $\omega$ varied within $3 \sigma$ allowed ranges as shown in Sec. IVA. The TBM and GR cases are excluded because, for the allowed values of $\theta_{12}$, they predict $\theta_{23}$ beyond the $3 \sigma$ range. The $3 \sigma$ limiting values of $\theta_{13}$ are marked by the solid lines, whereas the dashed lines indicate its best-fit value. The vertical and horizontal blue dot-dashed lines denote the $3 \sigma$ experimental limits of $\theta_{12}$ and $\theta_{23}$.

With the help of Eq. (28), one can translate any allowed point in the $\omega-\theta_{12}$ plane and the $\theta_{13}$ associated with it to a value of $z$, or equivalently $m_{0}$, when the solar and the atmospheric mass splittings are provided. For both the allowed mixing patterns $m_{0}$ varies over a very small range. This range is found to be $2.13 \mathrm{meV} \leq m_{0} \leq 3.10 \mathrm{meV}$ $\left(3.20 \mathrm{meV} \leq m_{0} \leq 4.42 \mathrm{meV}\right.$ ) for NSM (BM) when both

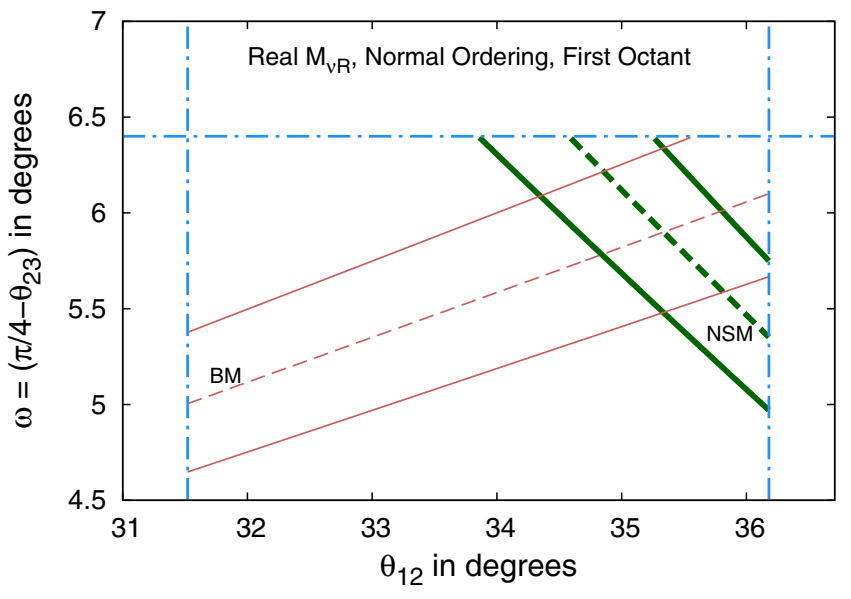

FIG. 1. $\omega=\left(\pi / 4-\theta_{23}\right)$-vs- $\theta_{12}$ plot for normal ordering. The $3 \sigma$ allowed range of $\sin \theta_{13}$ is marked by the solid lines whereas the dashed line indicates the best-fit value. Thin pink (thick green) lines denote the BM (NSM) case. The horizontal and vertical lines represent the data allowed $3 \sigma$ range. The first octant of $\theta_{23}$ is preferred since $\omega$ is positive always. Although $\omega$ is positive for TBM and GR mixing patterns its value lies beyond the $3 \sigma$ range. Best-fit values of atmospheric and solar mass splittings are taken. Inverted ordering is disallowed for $M_{\nu R}$ real.

mass splittings and all the three mixing angles are allowed to vary over their entire $3 \sigma$ ranges.

The salient features of the real $M_{\nu R}$ case are

(1) Only the normal ordering of neutrino masses is allowed.

(2) Only the first octant of $\theta_{23}$ is admissible.

(3) Type-I seesaw corrections is unable to make the TBM and GR mixing patterns consistent with the allowed ranges of the mixing angles.

(4) NSM and BM alternatives can produce solutions in agreement with the observed neutrino masses and mixing. The allowed ranges of lightest neutrino mass is very narrow.

\section{Complex $M_{\nu R}$}

Real $M_{\nu R}$ has several limitations viz. inverted ordering and $C P$-violation is forbidden. Moreover, TBM and GR mixing patterns cannot be included within the ambit of the model when $M_{\nu R}$ is real. In order to overcome these constraints, the general complex form of $M_{\nu R}$ leading to the type-I seesaw contribution $M^{\prime}$ furnished in Eq. (14) has to be considered. It is worth reminding ourselves that this choice introduces the complex phases $\phi_{1,2}$, while $x$ and $y$ can only be positive.

Thus, $M^{\prime}$ is no longer Hermitian. To retain the Hermitian nature, the combination $\left(M^{0}+M^{\prime}\right)^{\dagger}\left(M^{0}+M^{\prime}\right)$ is considered, among which $M^{0 \dagger} M^{0}$ and $\left(M^{0 \dagger} M^{\prime}+M^{\prime \dagger} M^{0}\right)$ are treated as the leading term and the perturbation at the lowest order, respectively. The unperturbed eigenvalues are given by $\left(m_{i}^{(0)}\right)^{2}$ and perturbation matrix is 


$$
\begin{aligned}
& \left(M^{0 \dagger} M^{\prime}+M^{\prime \dagger} M^{0}\right) \\
& =\frac{m_{D}^{2}}{m_{R}}\left(\begin{array}{ccc}
0 & 2 y m_{1}^{(0)} \cos \phi_{1} & y f\left(\phi_{1}\right) \\
2 y m_{1}^{(0)} \cos \phi_{1} & \sqrt{2} x m_{1}^{(0)} \cos \phi_{2} & -\frac{x}{\sqrt{2}} f\left(\phi_{2}\right) \\
y f^{*}\left(\phi_{1}\right) & -\frac{x}{\sqrt{2}} f^{*}\left(\phi_{2}\right) & \sqrt{2} x m_{3}^{(0)} \cos \phi_{2}
\end{array}\right),
\end{aligned}
$$

where

$$
f(\varphi) \equiv m^{+} \cos \varphi-i m^{-} \sin \varphi .
$$

The rest of the procedure is analogous to what was done in case of real $M_{\nu R}$ keeping in mind the discriminating factors of Eq. (31). Now, instead of Eqs. (18) and (19) of the real $M_{\nu R}$ case, the solar mixing obtained from Eq. (31) is given by

$$
\theta_{12}=\theta_{12}^{0}+\zeta, \quad \tan 2 \zeta=2 \sqrt{2} \frac{y}{x} \frac{\cos \phi_{1}}{\cos \phi_{2}}
$$

and

$$
\begin{aligned}
\sin \epsilon & =\frac{y \cos \phi_{1}}{\sqrt{y^{2} \cos ^{2} \phi_{1}+x^{2} \cos ^{2} \phi_{2} / 2}}, \\
\cos \epsilon & =\frac{x \cos \phi_{2} / \sqrt{2}}{\sqrt{y^{2} \cos ^{2} \phi_{1}+x^{2} \cos ^{2} \phi_{2} / 2}} \\
\tan \epsilon & =\frac{1}{2} \tan 2 \zeta .
\end{aligned}
$$

Table IV shows the allowed ranges of $\zeta$ and $\epsilon$ which depend on the mixing patterns. For all mixing alternatives $\cos \epsilon$ is found to be positive. Thus, from Eq. (34), $\phi_{2}$ must always lie in the first or fourth quadrants. For the different mixing patterns the ranges of $\phi_{1}$ are also given by that of $\epsilon$. When $\epsilon$ is positive (negative) then from the first relation contained in Eq. (34), it is evident that $\phi_{1}$ has to be in the first or fourth (second or third) quadrants. Using the results displayed in Table IV, one can infer that the first (second) option holds for the NSM (BM) patterns. In case of TBM and GR, $\epsilon$ varies over positive and negative values making both options equally admissible.

Applying degenerate perturbation theory the solar mass splitting attributed completely to the type-I seesaw contribution can be obtained from Eq. (31):

$$
\Delta m_{\text {solar }}^{2}=\sqrt{2} m_{1}^{(0)} \frac{m_{D}^{2}}{m_{R}} \sqrt{x^{2} \cos ^{2} \phi_{2}+8 y^{2} \cos ^{2} \phi_{1}}=\sqrt{2} m_{1}^{(0)} \frac{m_{D}^{2}}{m_{R}} \frac{x \cos \phi_{2}}{\cos 2 \zeta}=\sqrt{2} m_{1}^{(0)} \frac{m_{D}^{2}}{m_{R}} \frac{2 \sqrt{2} y \cos \phi_{1}}{\sin 2 \zeta} .
$$

In place of Eq. (23), one gets

$$
\left|\psi_{3}\right\rangle=\left(\begin{array}{c}
\kappa_{c}\left[\frac{\sin \epsilon}{\cos \phi_{1}} f\left(\phi_{1}\right) \cos \theta_{12}^{0}-\frac{\cos \epsilon}{\cos \phi_{2}} f\left(\phi_{2}\right) \sin \theta_{12}^{0}\right] / m^{+} \\
\frac{1}{\sqrt{2}}\left\{1-\kappa_{c}\left[\frac{\sin \epsilon}{\cos \phi_{1}} f\left(\phi_{1}\right) \sin \theta_{12}^{0}+\frac{\cos \epsilon}{\cos \phi_{2}} f\left(\phi_{2}\right) \cos \theta_{12}^{0}\right] / m^{+}\right\} \\
\frac{1}{\sqrt{2}}\left\{1+\kappa_{c}\left[\frac{\sin \epsilon}{\cos \phi_{1}} f\left(\phi_{1}\right) \sin \theta_{12}^{0}+\frac{\cos \epsilon}{\cos \phi_{2}} f\left(\phi_{2}\right) \cos \theta_{12}^{0}\right] / m^{+}\right\}
\end{array}\right),
$$

where

$$
\kappa_{c}=\frac{m_{D}^{2}}{m_{R} m^{-}} \sqrt{y^{2} \cos ^{2} \phi_{1}+x^{2} \cos ^{2} \phi_{2} / 2}
$$

Here Eq. (34) and the complex function $f\left(\phi_{1,2}\right)$ defined in Eq. (32) have been used. $\kappa_{c}$ is positive (negative) for NO (IO). Comparing Eq. (36) with the third column of Eq. (1) leads to

$$
\sin \theta_{13} \cos \delta=\kappa_{c} \sin \left(\epsilon-\theta_{12}^{0}\right)
$$

$$
\begin{aligned}
\sin \theta_{13} \sin \delta= & \kappa_{c} \frac{m^{-}}{m^{+} \cos \phi_{1} \cos \phi_{2}}\left[\sin \epsilon \sin \phi_{1} \cos \phi_{2} \cos \theta_{12}^{0}\right. \\
& \left.-\cos \epsilon \cos \phi_{1} \sin \phi_{2} \sin \theta_{12}^{0}\right]
\end{aligned}
$$

From Table IV, it is obvious that $\left(\epsilon-\theta_{12}^{0}\right)$ exists in the first (fourth) quadrant for the NSM (BM, TBM, and GR) mixing pattern. From Eq. (38), one can immediately conclude that for NSM (BM, TBM, and GR) case(s) $\delta$ remains in the first or fourth (second or third) quadrants in case of normal ordering. $\kappa_{c}$ changes sign for inverted ordering. Thus, the quadrants get modified accordingly. The different alternatives are furnished in Table V. There are two allowed quadrants of $\delta$ having $\sin \delta$ of opposite sign for any mixing option and ordering of neutrino masses. The sign of the right-hand-side of Eq. (39) governs the phases $\phi_{1,2}$ which in its turn decides the quadrants $C P$-phase $\delta$ out of the two allowed options. As already discussed, $\phi_{2}$ can be in either the first or fourth quadrants. The quadrant of $\phi_{1}$ 
TABLE V. The octant of $\theta_{23}$ and the quadrants of the $C P$-phase $\delta$ for different mixing patterns for both orderings of neutrino masses are exhibited.

\begin{tabular}{llclll}
\hline \hline & \multicolumn{2}{c}{ Normal ordering } & & \multicolumn{2}{c}{ Inverted ordering } \\
\cline { 2 - 3 } \cline { 5 - 6 } $\begin{array}{l}\text { Mixing } \\
\text { pattern }\end{array}$ & $\delta$ Quadrant & $\theta_{23}$ & Octant & $\delta$ Quadrant & $\theta_{23}$ Octant \\
\hline NSM & First/fourth & First & Second/third & Second \\
BM, TBM, & Second/third & First & First/fourth & Second \\
GR & & & & \\
\hline \hline
\end{tabular}

depends on the mixing pattern in such a manner that $\sin \phi_{1}$ can be of either sign. Therefore, the phases $\phi_{1}$ and $\phi_{2}$ can be chosen in a way such that $\sin \delta$ can acquire any particular sign. Thus, the two alternate quadrants of $\delta$ for every case in Table $\mathrm{V}$ are equally allowed in the model.

The type-I seesaw perturbative contribution to the atmospheric mixing angle can be obtained from Eq. (36) as

$$
\tan \omega=\frac{\sin \theta_{13} \cos \delta}{\tan \left(\epsilon-\theta_{12}^{0}\right)} .
$$

Let us recall that Eq. (38) relates $\delta$ and $\left(\epsilon-\theta_{12}^{0}\right)$ through $\kappa_{c}$. Thus, for all mixing alternatives $\theta_{23}$ always remains in first (second) octant for NO (IO). This is one of the most important results of the model as shown in Table V.

In the solar splitting expressed in Eq. (35), the factor of $m_{D}^{2} / m_{R}$ can be replaced in terms of $\kappa_{c}$. This together with Eq. (38) gives,

$$
\Delta m_{\text {solar }}^{2}=\frac{2 m^{-} m_{1}^{(0)} \sin \theta_{13} \cos \delta \cos \epsilon}{\sin \left(\epsilon-\theta_{12}^{0}\right) \cos 2 \zeta} .
$$

Predictions of the model can be extracted from Eqs. (40) and (41). The three mixing angles $\theta_{13}, \theta_{12}$, and $\theta_{23}$ are taken as inputs. Equation (40) determines a value of the
$C P$-violating phase $\delta$. With the help of these and the experimentally observed solar splitting the combination $m_{1}^{(0)} m^{-}$, or equivalently the variable $z$ can be calculated using Eq. (41) that fixes the lightest neutrino mass $m_{0}$. It may seem that arbitrarily large values of $m_{0}$, and hence $m_{1}^{(0)} m^{-}$, may be accounted for by tuning $\cos \delta$ to smaller and smaller values. However, this certainly is not the case. Experimental data necessitate $\omega=\left(\pi / 4-\theta_{23}\right)$ to be restricted within observed limits. As all other factors have ranges determined experimentally, Eq. (40) also puts lower and upper bounds on $\delta$. Subsequently, $m_{0}$ lies within a fixed range for any mixing pattern.

Figure 2 contains the $C P$-phase $\delta\left(\theta_{23}\right)$ as a function of the lightest neutrino mass $m_{0}$ for different mixing patterns as predicted by this model in the left (right) panel while the best-fit values of the various measured angles and mass splittings are used. The NSM, BM, TBM and GR are depicted by green solid, pink dashed, red dot-dashed, and violet dotted curves respectively. The thick (thin) curves of each kind indicate NO (IO). Normal and inverted orderings are always associated with the first and second octants of the atmospheric mixing angle $\theta_{23}$ respectively. For NSM case $\delta$ lies in the first (second) quadrant for normal (inverted) ordering, while for the rest of the mixing options it is in the second (first) quadrant. For inverted neutrino mass ordering, $|\delta|$ remains close to $\pi / 2$ for the complete range of $m_{0}$. The $C P$-phase $\delta$ lies near $\pi / 2$ for normal ordering for $m_{0}$ larger than around $0.05 \mathrm{eV}$.

From Table $\mathrm{V}$, it is evident that if $\delta$ is a solution for some $m_{0}$ then by properly choosing alternate values of the phases $\phi_{1,2}$ appearing in $M_{\nu R}$ one can also obtain a second solution with the phase $-\delta$. This mirror set of solutions are not shown in Fig. 2. The preliminary data presented by the T2K [21] and NOVA [22] collaborations can be considered as primary hint of normal ordering associated with $\delta \sim-\pi / 2$. The consistency of this model with these observations is clearly visible from Fig. 2 with $\delta \sim-\pi / 2$ favoring $m_{0}$ in the

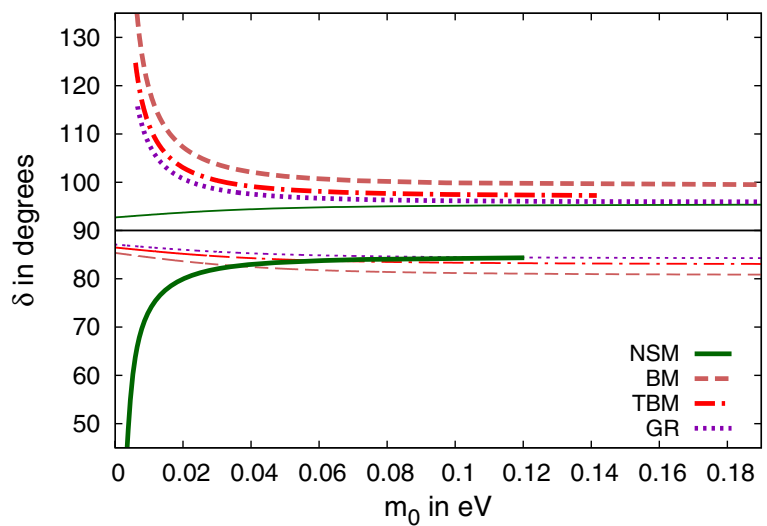

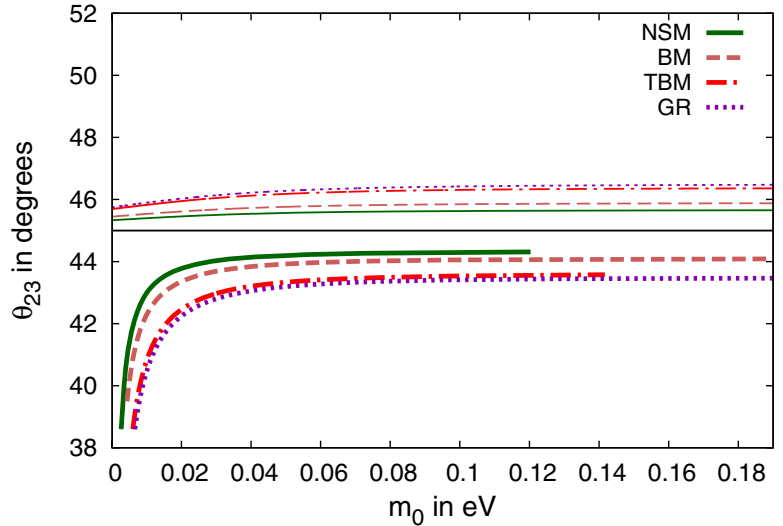

FIG. 2. In the left (right) panel, the $C P$-phase $\delta\left(\theta_{23}\right)$ predicted by this model is plotted as a function of the lightest neutrino mass $m_{0}$ for all the four mixing patterns when the best-fit values of the data are taken as input. The NSM, BM, TBM and GR mixing alternatives are represented by the green solid, pink dashed, red dot-dashed, and violet dotted curves respectively. Thick (thin) curves of each kind denote NO (IO). 
quasidegenerate regime, i.e., $m_{0} \geq \mathcal{O}(0.05 \mathrm{eV})$, for normal ordering. If this result is determined with better accuracy in the future analysis then the model will predict neutrino masses to be in a range that ongoing experiments are capable of probing $[23,24]$.

These interrelationships between the octant of $\theta_{23}$, the quadrant of the $C P$-violating phase $\delta$, and the neutrino mass ordering provide a clear set of correlations characteristic of this $A 4$ based model. In the model the corrections to the three neutrino mixing angles and $\Delta m_{\text {solar }}^{2}$ all have a common origin - the type-I seesaw. As a result these parameters get correlated. Such interrelationships are specific to this model. Although the model has a large number of parameters, only this correlated region of the parameter space allowed by neutrino mass and mixing data leads to testable predictions in Table V.

\section{CONCLUSIONS}

In this paper, an $A 4$ based seesaw model for neutrino masses and mixing has been proposed. The flavor quantum numbers suitable for the model are assigned to the leptons and the scalars. The Lagrangian is inclusive of all the symmetry conserving terms. No soft breaking of symmetry is entertained. The Yukawa couplings induce the charged lepton masses, Dirac and Majorana masses for the left- and right-handed neutrinos after the symmetry is broken spontaneously. Neutrino masses are produced by a combined effect of both type-I and type-II seesaw terms present in the Lagrangian of which the former can be thought of to be a small correction. The type-II seesaw dominant contribution is associated with the atmospheric mass splitting, no solar splitting, keeps $\theta_{23}=\pi / 4$, and $\theta_{13}=0$ and $\theta_{12}$ can be given any preferred value. In particular, this model is scrutinized in context of tribimaximal, bimaximal, golden ratio, and 'no solar mixing' patterns. The contribution of type-I seesaw can be treated as a perturbation that generates the solar splitting and tunes the mixing angles to values in agreement with the global fits. As a corollary, a correlation between the octants of $\theta_{23}$ and neutrino mass ordering followed - the first (second) octant is allowed for normal (inverted) ordering of neutrino mass. The model has several testable predictions including that of the $C P$-phase $\delta$, relationships between mixing angles and mass splittings. Moreover, inverted ordering got associated with nearmaximal $C P$-phase $\delta$ and arbitrarily small neutrino masses are allowed. In the case of normal ordering, $\delta$ can vary over a larger range and maximality is accomplished in the quasidegenerate regime. The lightest neutrino mass has to be at least a few meV for this case.

\section{ACKNOWLEDGMENTS}

I acknowledge support from CSIR, India, during the initial stage of this work at the University of Calcutta. I also thank Professor Amitava Raychaudhuri for his valuable insights and comments at various stages of this endeavor.

\section{APPENDIX A: THE GROUP A4}

$A 4$ is the even permutation group of four objects having 12 elements and two generators $S$ and $T$ satisfying the property $S^{2}=T^{3}=(S T)^{3}=\mathbb{I}$. It has four inequivalent irreducible representations viz. one 3-dimensional representation and three 1-dimensional representations, namely, $1,1^{\prime}$ and $1^{\prime \prime}$. These three dimension-one representations are singlets under $S$, whereas they transform as $1, \omega$, and $\omega^{2}$, respectively, under the action of $T$, with $\omega$ being a cube root of unity. Therefore, it is apparent that $1^{\prime} \times 1^{\prime \prime}=1$. The pertinent form of the generators $S$ and $T$ acting on the threedimensional representations are given by ${ }^{12}$

$S=\left(\begin{array}{ccc}1 & 0 & 0 \\ 0 & -1 & 0 \\ 0 & 0 & -1\end{array}\right) \quad$ and $\quad T=\left(\begin{array}{lll}0 & 1 & 0 \\ 0 & 0 & 1 \\ 1 & 0 & 0\end{array}\right)$

It is imperative to note that the product rule for the threedimensional representation is

$$
3 \otimes 3=1 \oplus 1^{\prime} \oplus 1^{\prime \prime} \oplus 3 \oplus 3 .
$$

When two triplets of $A 4$ given by $3_{a} \equiv a_{i}$ and $3_{b} \equiv b_{i}$, with $i=1,2,3$, are combined according to Eq. (A2), then the resulting triplets can be represented by $3_{c} \equiv c_{i}$ and $3_{d} \equiv d_{i}$, where

$$
\begin{aligned}
c_{i} & =\left(\frac{a_{2} b_{3}+a_{3} b_{2}}{2}, \frac{a_{3} b_{1}+a_{1} b_{3}}{2}, \frac{a_{1} b_{2}+a_{2} b_{1}}{2}\right), \\
\text { or, } \quad c_{i} & \equiv \alpha_{i j k} a_{j} b_{k}, \\
d_{i} & =\left(\frac{a_{2} b_{3}-a_{3} b_{2}}{2}, \frac{a_{3} b_{1}-a_{1} b_{3}}{2}, \frac{a_{1} b_{2}-a_{2} b_{1}}{2}\right), \\
\text { or, } \quad d_{i} & \equiv \beta_{i j k} a_{j} b_{k}, \quad(i, j, k, \text { are cyclic })
\end{aligned}
$$

and the $1,1^{\prime}$ and $1^{\prime \prime}$ so obtained can be scripted as

$$
\begin{aligned}
1 & =a_{1} b_{1}+a_{2} b_{2}+a_{3} b_{3} \equiv \rho_{1 i j} a_{i} b_{j}, \\
1^{\prime} & =a_{1} b_{1}+\omega^{2} a_{2} b_{2}+\omega a_{3} b_{3} \equiv \rho_{3 i j} a_{i} b_{j}, \\
1^{\prime \prime} & =a_{1} b_{1}+\omega a_{2} b_{2}+\omega^{2} a_{3} b_{3} \equiv \rho_{2 i j} a_{i} b_{j} .
\end{aligned}
$$

The group is studied in extensive detail in $[9,10]$.

\footnotetext{
${ }^{12}$ This choice of basis has the generator $S$ diagonal. One can equivalently perform an analogous analysis in a basis in which the generator $T$ is diagonal. Needless to mention that the two bases are related by some unitary basis transformation.
} 


\section{APPENDIX B: MINIMIZATION OF THE SCALAR POTENTIAL}

Some detailed analysis of the nature of the scalar potential is presented in this Appendix. The conditions that have to be satisfied by the parameters of the potential so that the vevs acquire the values considered in the model are extracted. The conditions so obtained guarantee the potential is locally minimized by those choices. To confirm if those choices concur with the global minimum is beyond the scope of this work. ${ }^{13}$

The fields cataloged in Table III are comprised of scalars having lepton numbers as well as $A 4, S U(2)_{L}$, and $U(1)_{Y}$ charges. The scalar potential must be of the most general quartic nature conserving all the symmetries under consideration. Thus, all the terms allowed by the symmetries are included in the discussion below. Verification of $S U(2)_{L}, U(1)_{Y}$ and lepton number are familiar exercises. $A 4$ invariance requires elaborate discussion as presented in the following section.

\section{A4 conserving terms: Notations and general principles}

Let us summarize a few salient features of this model to fix the notations to be followed for the A4-invariant terms. As already noted, the scalar spectrum has fields transforming as $1,1^{\prime}, 1^{\prime \prime}$, and 3 under $A 4$. One has to consider all the combinations of these fields up to quartics that can yield $A 4$ invariants. The product rules for $1,1^{\prime}$ and $1^{\prime \prime}$ are easy, but that for the triplets of $A 4$ needs to be emphasized. If there are two $A 4$ triplet fields $A \equiv\left(a_{1}, a_{2}, a_{3}\right)^{T}$ and $B \equiv$ $\left(b_{1}, b_{2}, b_{3}\right)^{T}$ where $a_{i}, b_{i}$ may possess $S U(2)_{L} \times U(1)_{Y}$ transformation properties that are not considered for the time being in the immediate course of discussion. As furnished in Eq. (A2), one can combine $A$ and $B$ to obtain

$$
3_{A} \otimes 3_{B}=1 \oplus 1^{\prime} \oplus 1^{\prime \prime} \oplus 3 \oplus 3 .
$$

For notational simplicity, let us denote the irreducible representations on the right-hand side by $O_{1}(A, B)$, $\mathrm{O}_{2}(A, B), O_{3}(A, B), T_{s}(A, B)$ and $T_{a}(A, B)$, respectively, where, as already noted, in Eqs. (A3) and (A4),

$$
\begin{aligned}
& O_{1}(A, B) \equiv 1=a_{1} b_{1}+a_{2} b_{2}+a_{3} b_{3} \equiv \rho_{1 i j} a_{i} b_{j}, \\
& O_{2}(A, B) \equiv 1^{\prime}=a_{1} b_{1}+\omega^{2} a_{2} b_{2}+\omega a_{3} b_{3} \equiv \rho_{3 i j} a_{i} b_{j}, \\
& O_{3}(A, B) \equiv 1^{\prime \prime}=a_{1} b_{1}+\omega a_{2} b_{2}+\omega^{2} a_{3} b_{3} \equiv \rho_{2 i j} a_{i} b_{j},
\end{aligned}
$$

and

$$
\begin{aligned}
& T_{s}(A, B) \equiv 3=\left(\frac{a_{2} b_{3}+a_{3} b_{2}}{2}, \frac{a_{3} b_{1}+a_{1} b_{3}}{2}, \frac{a_{1} b_{2}+a_{2} b_{1}}{2}\right)^{T}, \\
& T_{a}(A, B) \equiv 3=\left(\frac{a_{2} b_{3}-a_{3} b_{2}}{2}, \frac{a_{3} b_{1}-a_{1} b_{3}}{2}, \frac{a_{1} b_{2}-a_{2} b_{1}}{2}\right)^{T} .
\end{aligned}
$$

It is worth noting that $O_{3}\left(A^{\dagger}, A\right)=\left[O_{2}\left(A^{\dagger}, A\right)\right]^{\dagger}$ and $T_{a}(A, A)=0$.

The scalar potential can be formulated implementing this notation and keeping in mind that the scalar sector of this model is devoid of any field which is invariant under all the symmetries under consideration. Therefore the scalar potential will contain terms of the following kind (only $A 4$ properties are exhibited):

(i) Quadratic: $W^{\dagger} W$,

(ii) Cubic: $X_{i} X_{j}^{\prime} X_{k}^{\prime \prime}, X_{i} X_{j} X_{k}, X_{i}^{\prime} X_{j}^{\prime} X_{k}^{\prime}, X_{i}^{\prime \prime} X_{j}^{\prime \prime} X_{k}^{\prime \prime}, O_{1}\left(Y_{i}, Y_{j}\right) X_{k}, O_{2}\left(Y_{i}, Y_{j}\right) X_{k}^{\prime \prime}, O_{3}\left(Y_{i}, Y_{j}\right) X_{k}^{\prime}$,

(iii) Quartic: $\left(W_{i}^{\dagger} W_{i}\right)\left(W_{j}^{\dagger} W_{j}\right),\left(X_{i} X_{j}\right)\left(X_{k} X_{l}\right),\left(X_{i} X_{j}\right)\left(X_{k}^{\prime} X_{l}^{\prime \prime}\right), \quad\left(X_{i}^{\prime} X_{j}^{\prime \prime}\right)\left(X_{k}^{\prime} X_{l}^{\prime \prime}\right),\left(X_{i}^{\prime} X_{j}^{\prime}\right)\left(X_{k}^{\prime} X_{l}\right), \quad\left(X_{i}^{\prime \prime} X_{j}^{\prime \prime}\right)\left(X_{k}^{\prime \prime} X_{l}\right)$, $O_{1}\left(Y_{i}, Y_{j}\right) X_{k} X_{l}, O_{1}\left(Y_{i}, Y_{j}\right) X_{k}^{\prime} X_{l}^{\prime \prime}, \quad O_{2}\left(Y_{i}, Y_{j}\right) X_{k}^{\prime} X_{l}^{\prime}, \quad O_{2}\left(Y_{i}, Y_{j}\right) X_{k} X_{l}^{\prime \prime}, \quad O_{3}\left(Y_{i}, Y_{j}\right) X_{k}^{\prime \prime} X_{l}^{\prime \prime}, O_{3}\left(Y_{i}, Y_{j}\right) X_{k} X_{l}^{\prime}$, $O_{1}\left(Y_{i}, Y_{j}\right) O_{1}\left(Y_{k}, Y_{l}\right), \quad O_{2}\left(Y_{i}, Y_{j}\right)^{\dagger} O_{2}\left(Y_{k}, Y_{l}\right), \quad O_{3}\left(Y_{i}, Y_{j}\right)^{\dagger} O_{3}\left(Y_{k}, Y_{l}\right), \quad O_{2}\left(Y_{i}, Y_{j}\right) O_{3}\left(Y_{k}, Y_{l}\right), \quad O_{1}\left(T_{s}\left(Y_{i}, Y_{j}\right)\right.$, $\left.T_{s}\left(Y_{k}, Y_{l}\right)\right), O_{1}\left(T_{s}\left(Y_{i}, Y_{j}\right), T_{a}\left(Y_{k}, Y_{l}\right)\right), O_{1}\left(T_{a}\left(Y_{i}, Y_{j}\right), T_{a}\left(Y_{k}, Y_{l}\right)\right) . O_{1}\left(T_{s}\left(Y_{i}, Y_{j}\right), Y_{k}\right) X_{l}, O_{2}\left(T_{s}\left(Y_{i}, Y_{j}\right), Y_{k}\right) X_{l}^{\prime \prime}$, $O_{3}\left(T_{s}\left(Y_{i}, Y_{j}\right), Y_{k}\right) X_{l}^{\prime}, O_{1}\left(T_{a}\left(Y_{i}, Y_{j}\right), Y_{k}\right) X_{l}, O_{2}\left(T_{a}\left(Y_{i}, Y_{j}\right), Y_{k}\right) X_{l}^{\prime \prime}, O_{3}\left(T_{a}\left(Y_{i}, Y_{j}\right), Y_{k}\right) X_{l}^{\prime}$.

\footnotetext{
${ }^{13}$ As an example, one can take a look at [25], where a comparatively simpler scenario, consisting of an $A 4$ triplet composed of three $S U(2)_{L}$ doublet scalar or, in other words, an $A 4$ symmetric three Higgs doublet model (3HDM), was analyzed in terms of the global minimization of the scalar potential. In [26], it is shown that alignment follows as a natural consequence when the vevs acquire the configurations corresponding to those global minima. Three Higgs doublets symmetric under the A4 group has been vividly discussed in [27]. A model for leptons using an $A 4$ symmetric $3 \mathrm{HDM}$ can be found in [28].
}

Here $W$ is any field, $X, X^{\prime}$, and $X^{\prime \prime}$ represent generic fields transforming as $1,1^{\prime}$, and $1^{\prime \prime}$ under $A 4$ while $Y$ happens to be generic $A 4$ triplet field. The invariants constructed by using $X^{\dagger}, X^{\prime \dagger}, X^{\prime \prime}$, and $Y^{\dagger}$ are not listed separately.

Owing to the large number of scalars in the model-e.g., $S U(2)_{L}$ singlets, doublets, and triplets- the scalar potential consists of many terms. In order to simplify the discussion, cubic terms in the fields are excluded and all the couplings are taken to be real. The antisymmetric triplet arising from 
the combination of two $A 4$ triplets i.e., the terms denoted by $T_{a}$ in Eq. (B3) are not included in the potential throughout for ease of calculation. The potential is studied piecewise: (a) consisting of terms that arise from a combination of fields belonging to same $S U(2)_{L}$ sector, and (b) comprising terms obtained by combining scalars of different $S U(2)_{L}$ sectors. The vev of the $S U(2)_{L}$ singlets giving rise to the righthanded neutrino mass are larger than the vev of the other scalar fields. Thus, in the latter category the combinations of $S U(2)_{L}$ singlets with the doublets and triplets of $S U(2)_{L}$ are considered, whereas, doublet-triplet inter-sector terms are dropped owing to the smallness of the triplet vev responsible for the left-handed Majorana neutrino mass. Also the electroweak precision measurements put a stringent bound on the triplet vev compelling it to be very small.

\section{2. $S U(2)_{L}$ singlet sector}

The $S U(2)_{L}$ singlet scalar sector consists of three $A 4$ triplets $\hat{\Delta}_{p}^{R}$ with $p=a, b, c$ denoting each one of them. These three triplets possess identical quantum numbers, their vev being the only discriminating criterion. Also there are three more fields viz. $\Delta_{1}^{R}, \Delta_{2}^{R}$ and $\Delta_{3}^{R}$ transforming as 1 , $1^{\prime}$ and $1^{\prime \prime}$ under $A 4$. From Eq. (B1) we can see that two same $\hat{\Delta}_{p}^{R}$ triplets can combine to produce several $A 4$ irreducible representations. For notational simplicity let us define:

$$
\begin{aligned}
O_{1 p}^{s s} & \equiv O_{1}\left(\hat{\Delta}_{p}^{R^{\dagger}}, \hat{\Delta}_{p}^{R}\right) ; \quad O_{2 p}^{s s} \equiv O_{2}\left(\hat{\Delta}_{p}^{R^{\dagger}}, \hat{\Delta}_{p}^{R}\right) ; \\
T_{s p}^{s s} & \equiv T_{s}\left(\hat{\Delta}_{p}^{R}, \hat{\Delta}_{p}^{R}\right), \quad(p=a, b, c) .
\end{aligned}
$$

Using two different triplets $\hat{\Delta}_{p}^{R}$ and $\hat{\Delta}_{q}^{R}$ where $p \neq q$ analogous combinations can be defined:

$$
\begin{aligned}
& \hat{O}_{1 p q}^{s s} \equiv O_{1}\left(\hat{\Delta}_{p}^{R^{\dagger}}, \hat{\Delta}_{q}^{R}\right) ; \quad \hat{O}_{2 p q}^{s s} \equiv O_{2}\left(\hat{\Delta}_{p}^{R \dagger}, \hat{\Delta}_{q}^{R}\right) ; \\
& \hat{T}_{s p q}^{s s} \equiv T_{s}\left(\hat{\Delta}_{p}^{R}, \hat{\Delta}_{q}^{R}\right), \quad(p, q=a, b, c \quad \text { and } \quad p \neq q) .
\end{aligned}
$$

Generically, it is convenient to use $\tilde{O}_{i p}$ or $\tilde{T}_{s p}$ if the second triplet in the argument is replaced by its Hermitian conjugate. As an example,

$$
\begin{aligned}
& \tilde{O}_{1 p}^{s s} \equiv O_{1}\left(\hat{\Delta}_{p}^{R^{\dagger}}, \hat{\Delta}_{p}^{R^{\dagger}}\right), \quad \tilde{O}_{2 p}^{s s} \equiv O_{2}\left(\hat{\Delta}_{p}^{R^{\dagger}}, \hat{\Delta}_{p}^{R^{\dagger}}\right), \\
& \tilde{O}_{3 p}^{s s} \equiv O_{3}\left(\hat{\Delta}_{p}^{R \dagger}, \hat{\Delta}_{p}^{R \dagger}\right) \quad \text { and } \quad \tilde{T}_{s p}^{s s} \equiv T_{s}\left(\hat{\Delta}_{p}^{R}, \hat{\Delta}_{p}^{R^{\dagger}}\right),
\end{aligned}
$$

One can also consider

$$
\begin{aligned}
& \tilde{O}_{1 p q}^{s s} \equiv O_{1}\left(\hat{\Delta}_{p}^{R^{\dagger}}, \hat{\Delta}_{q}^{R^{\dagger}}\right), \quad \tilde{O}_{2 p q}^{s s} \equiv O_{2}\left(\hat{\Delta}_{p}^{R^{\dagger}}, \hat{\Delta}_{q}^{R^{\dagger}}\right), \\
& \tilde{O}_{3 p q}^{s s} \equiv O_{3}\left(\hat{\Delta}_{p}^{R^{\dagger}}, \hat{\Delta}_{q}^{R^{\dagger}}\right) .
\end{aligned}
$$

Also the following combinations are required:

$$
\begin{aligned}
& \mathcal{O}_{1 p}^{s s} \equiv O_{1}\left(\hat{\Delta}_{p}^{R}, T_{s p}^{s s \dagger}\right), \quad \mathcal{O}_{2 p}^{s s} \equiv O_{2}\left(\hat{\Delta}_{p}^{R}, T_{s p}^{s s \dagger}\right), \\
& \mathcal{O}_{3 p}^{s s} \equiv O_{3}\left(\hat{\Delta}_{p}^{R}, T_{s p}^{s s \dagger}\right), \quad(p=a, b, c) \text {. }
\end{aligned}
$$

The $A 4$ singlets $\Delta_{i}^{R}(i=1,2,3)$ can be combined to yield

$$
Q_{i}^{s s} \equiv \Delta_{i}^{R \dagger} \Delta_{i}^{R}, \quad(i=1,2,3) .
$$

Needless to mention that such terms are singlets of all the symmetries under consideration.

Having devised the essential notations one can write the most general scalar potential for the $S U(2)_{L}$ singlet sector of this model as

$$
\begin{aligned}
V_{\text {singlet }}= & \sum_{i=1}^{3} m_{\Delta_{i}^{R}}^{2} Q_{i}^{s s}+\sum_{p=a}^{c} m_{\hat{\Delta}_{p}^{R}}^{2} O_{1 p}^{s s}+\left[\sum_{p \neq q ; p, q=a}^{c} m_{\hat{\Delta}_{p q}^{R}}^{2} \hat{O}_{1 p q}^{s s}+\text { all possible permutations }\right] \\
& +\frac{1}{2} \sum_{i=1}^{3} \lambda_{1 i}^{s}\left[Q_{i}^{s s}\right]^{2}+\frac{1}{2} \sum_{k<j ; k \neq j ; k=1}^{2} \sum_{j=2}^{3} \lambda_{2 j k}^{s}\left\{Q_{j}^{s s} Q_{k}^{s s}\right\}+\frac{1}{2} \sum_{p=a}^{c} \lambda_{3 p}^{s}\left\{\left[O_{1 p}^{s s}\right]^{2}+\left(O_{2 p}^{s s}\right)^{\dagger} O_{2 p}^{s s}+O_{1 p}\left(T_{s p}^{s s}, T_{s p}^{s s \dagger}\right)\right\} \\
& +\sum_{p \neq q ; p, q=a}^{c} \lambda_{3 p q}^{s}\left\{\left[\hat{O}_{1 p q}^{s s}\right]^{2}+\left(\hat{O}_{2 p q}^{s s}\right)^{\dagger} \hat{O}_{2 p q}^{s s}+\text { H.c. }\right\}+\frac{1}{2} \sum_{p \neq q ; p, q=a}^{c} \tilde{\lambda}_{3 p q}^{s}\left\{\left(\hat{O}_{1 p q}^{s s}\right)^{\dagger} \hat{O}_{1 p q}^{s s}+O_{1}\left(\hat{T}_{s p q}^{s s}, \hat{T}_{s p q}^{s s \dagger}\right)\right\} \\
& +\sum_{p=a}^{c} \sum_{i=1}^{3}\left[\frac{1}{2} \lambda_{4 i p}^{s}\left(Q_{i}^{s s} O_{1 p}^{s s}\right)\right]+\sum_{i=1}^{3} \sum_{p \neq q ; p, q=a}^{c} \lambda_{4 i p q}^{s}\left[\left(Q_{i}^{s s} \hat{O}_{1 p q}^{s s}\right)+\text { H.c. }\right]+\frac{1}{2} \sum_{p=a}^{c} \lambda_{5 p}^{s}\left(\mathcal{O}_{1 p}^{s s} \Delta_{1}^{R}+\text { H.c. }\right) \\
& +\sum_{p \neq q ; p, q=a}^{c} \lambda_{5 p q}^{s}\left[\left\{\left(\Delta_{1}^{R} O_{1}\left(\hat{\Delta}_{p}^{R}, \hat{T}_{s p q}^{s s \dagger}\right)+\left(\Delta_{1}^{R} O_{1}\left(\hat{\Delta}_{q}^{R}, \hat{T}_{s p q}^{s s \dagger}\right)\right\}+\text { H.c. }\right]\right.\right. \\
& +\frac{1}{2} \sum_{p=a}^{c} \lambda_{6 p}^{s}\left(\mathcal{O}_{3 p}^{s s} \Delta_{2}^{R}+\text { H.c. }\right)+\sum_{p \neq q ; p, q=a}^{c} \lambda_{6 p q}^{s}\left[\left\{\left(\Delta_{2}^{R} O_{3}\left(\hat{\Delta}_{p}^{R}, \hat{T}_{s p q}^{s s \dagger}\right)+\left(\Delta_{2}^{R} O_{3}\left(\hat{\Delta}_{q}^{R}, \hat{T}_{s p q}^{s s \dagger}\right)\right\}+\text { H.c. }\right]\right.\right.
\end{aligned}
$$




$$
\begin{aligned}
& +\frac{1}{2} \sum_{p=a}^{c} \lambda_{7 p}^{s}\left(\mathcal{O}_{2 p}^{s s} \Delta_{3}^{R}+\text { H.c. }\right)+\sum_{p \neq q ; p, q=a}^{c} \lambda_{7 p q}^{s}\left[\left\{\left(\Delta_{3}^{R} O_{2}\left(\hat{\Delta}_{p}^{R}, \hat{T}_{s p q}^{s \dagger \dagger}\right)+\left(\Delta_{3}^{R} O_{2}\left(\hat{\Delta}_{q}^{R}, \hat{T}_{s p q}^{s \dagger \dagger}\right)\right\}+\text { H.c. }\right]\right.\right. \\
& +\sum_{p=a}^{c} \sum_{i=1}^{3} \lambda_{8 i p}^{s}\left(\Delta_{i}^{R 2} \tilde{O}_{i p}+\text { H.c. }\right)+\sum_{p \neq q ; p, q=a}^{c} \sum_{i=1}^{3} \lambda_{8 i p q}^{s}\left(\Delta_{i}^{R 2} \tilde{O}_{i p q}+\text { H.c. }\right) \\
& +\sum_{p=a}^{c}\left[\lambda_{91 p}^{s} \Delta_{2}^{R} \Delta_{3}^{R} \tilde{O}_{1 p}+\lambda_{92 p}^{s} \Delta_{1}^{R} \Delta_{3}^{R} \tilde{O}_{2 p}+\lambda_{93 p}^{s} \Delta_{1}^{R} \Delta_{2}^{R} \tilde{O}_{3 p}+\text { H.c. }\right] \\
& +\sum_{p \neq q ; p, q=a}^{c}\left[\lambda_{91 p q}^{s} \Delta_{2}^{R} \Delta_{3}^{R} \tilde{O}_{1 p q}+\lambda_{92 p q}^{s} \Delta_{1}^{R} \Delta_{3}^{R} \tilde{O}_{2 p q}+\lambda_{93 p q}^{s} \Delta_{1}^{R} \Delta_{2}^{R} \tilde{O}_{3 p q}+\text { H.c. }\right] .
\end{aligned}
$$

Here $\lambda_{3 p}^{s}, \lambda_{3 p q}^{s}$ and $\tilde{\lambda}_{3 p q}^{s}$ are taken as the common coefficient of the different $A 4$ invariants generated by combining two $\hat{\Delta}^{R}$ and two $\left(\hat{\Delta}^{R}\right)^{\dagger}$ fields. Similar policy will be adopted for the fields with other $S U(2)_{L}$ properties.

\section{3. $S U(2)_{L}$ doublet sector}

The $S U(2)_{L}$ doublet scalar precinct consists of the two fields $\Phi$ and $\eta$ transforming as 3 and 1 of $A 4$ respectively. Opposite hypercharges are assigned to $\Phi$ and $\eta$. The $A 4$ triplet $\Phi$ combinations are denoted as

$$
\begin{aligned}
O_{1}^{d d} & \equiv O_{1}\left(\Phi^{\dagger}, \Phi\right) ; \quad O_{2}^{d d} \equiv O_{2}\left(\Phi^{\dagger}, \Phi\right) ; \\
T_{s}^{d d} & \equiv T_{s}(\Phi, \Phi),
\end{aligned}
$$

and that of the $A 4$ singlet $\eta$ are

$$
Q_{\eta}^{d d} \equiv \eta^{\dagger} \eta
$$

The potential for the $S U(2)_{L}$ doublet sector is given by

$$
\begin{aligned}
V_{\text {doublet }}= & m_{\eta}^{2} Q_{\eta}^{d d}+m_{\Phi}^{2} O_{1}^{d d}+\frac{1}{2} \lambda_{1}^{d}\left[Q_{\eta}^{d d}\right]^{2} \\
& +\frac{1}{2} \lambda_{2}^{d}\left\{\left[O_{1}^{d d}\right]^{2}+\left\{O_{2}^{d d}\right\}^{\dagger} O_{2}^{d d}\right. \\
& \left.+O_{1}\left(T_{s}^{d d}, T_{s}^{d d \dagger}\right)\right\}+\frac{1}{2} \lambda_{3}^{d}\left[Q_{\eta}^{d d} O_{1}^{d d}\right] .
\end{aligned}
$$

\section{4. $S U(2)_{L}$ triplet sector}

The $S U(2)_{L}$ triplet sector is comprised of five fields. There are two $A 4$ triplets $\hat{\Delta}_{a}^{L}$ and $\hat{\Delta}_{b}^{L}$ together with the fields the $\Delta_{1}^{L}, \Delta_{2}^{L}$ and $\Delta_{3}^{L}$ transforming as $1,1^{\prime}, 1^{\prime \prime}$ of $A 4$ respectively.

It is useful to define:

$$
\begin{aligned}
O_{1 n}^{t t} & \equiv O_{1}\left(\hat{\Delta}_{n}^{L \dagger}, \hat{\Delta}_{n}^{L}\right) ; \quad O_{2 n}^{t t} \equiv O_{2}\left(\hat{\Delta}_{n}^{L \dagger}, \hat{\Delta}_{n}^{L}\right) ; \\
T_{s n}^{t t} & \equiv T_{s}\left(\hat{\Delta}_{n}^{L}, \hat{\Delta}_{n}^{L}\right), \quad(n=a, b),
\end{aligned}
$$

$\hat{O}_{1 n l}^{t t} \equiv O_{1}\left(\hat{\Delta}_{n}^{L \dagger}, \hat{\Delta}_{l}^{L}\right) ; \quad \hat{O}_{2 n l}^{t t} \equiv O_{2}\left(\hat{\Delta}_{n}^{L \dagger}, \hat{\Delta}_{l}^{L}\right) ;$

$\hat{O}_{3 n l}^{t t} \equiv O_{3}\left(\hat{\Delta}_{n}^{L \dagger}, \hat{\Delta}_{l}^{L}\right) ; \quad \hat{T}_{s n l}^{t t} \equiv T_{s}\left(\hat{\Delta}_{n}^{L}, \hat{\Delta}_{l}^{L}\right)$,

$(n, l=a, b \quad$ and $\quad n \neq l)$,

$$
Q_{i}^{t t} \equiv \Delta_{i}^{L \dagger} \Delta_{i}^{L}, \quad(i=1,2,3)
$$

and

$$
\begin{aligned}
\mathcal{O}_{\gamma n}^{t t} & \equiv O_{\gamma}\left(\hat{\Delta}_{n}^{L}, T_{s n}^{t t \dagger}\right) ; \quad \mathcal{O}_{\gamma n l}^{t t} \equiv O_{\gamma}\left(\hat{\Delta}_{n}^{L}, \hat{T}_{s l}^{t t \dagger}\right), \\
(\gamma & =1,2,3) \quad \text { and } \quad(n, l=a, b), \\
\tilde{O}_{j n}^{t t} & \equiv O_{j}\left(\hat{\Delta}_{n}^{L \dagger}, \hat{\Delta}_{n}^{L \dagger}\right) ; \quad \tilde{O}_{j n l}^{t t} \equiv O_{j}\left(\hat{\Delta}_{n}^{L \dagger}, \hat{\Delta}_{l}^{L \dagger}\right), \\
(j & =1,2,3) \quad \text { and } \quad(n, l=a, b \quad \text { and } \quad n \neq l) .
\end{aligned}
$$

The scalar potential for this sector:

$$
\begin{aligned}
V_{\text {triplet }}= & \sum_{i=1}^{3} m_{\Delta_{i}^{L}}^{2} Q_{i}^{t t}+\sum_{n=a}^{b} m_{\hat{\Delta}_{n}^{L}}^{2} O_{1 n}^{t t}+\left(\sum_{n \neq l ; n, l=a}^{b} m_{\hat{\Delta}_{n l}^{L}}^{2} \hat{O}_{1 n l}^{t t}+\text { all possible permutations }\right) \\
& +\frac{1}{2} \sum_{i=1}^{3} \lambda_{1_{i}}^{t}\left[Q_{i}^{t t}\right]^{2}+\frac{1}{2} \sum_{k<j, k=1}^{2} \sum_{j=2}^{3} \lambda_{2 j k}^{t} Q_{j}^{t t} Q_{k}^{t t}+\frac{1}{2} \sum_{n=a}^{b} \lambda_{3 n}^{t}\left\{\left[O_{1 n}^{t t}\right]^{2}+\left\{O_{2 n}^{t t}\right\}^{\dagger} O_{2 n}^{t t}+O_{1}\left(T_{s n}^{t t}, T_{s n}^{t t \dagger}\right)\right\} \\
& \left.+\frac{1}{2} \sum_{n \neq l ; n, l=a}^{b} \lambda_{3 n l}^{t}\left\{\left[\hat{O}_{1 n l}^{t t}\right]^{2}+\left\{\hat{O}_{2 n l}^{t t}\right\}^{\dagger} \hat{O}_{2 n l}^{t t}+\text { H.c. }\right\}+\frac{1}{2} \sum_{n \neq l ; n, l=a}^{b} \tilde{\lambda}_{3 n l}^{t}\left\{\left[\hat{O}_{1 n l}^{t t}\right]^{\dagger} \hat{O}_{1 n l}^{t t}\right]+O_{1}\left(\hat{T}_{s n}^{t t}, \hat{T}_{s n}^{t t \dagger}\right)\right\}
\end{aligned}
$$




$$
\begin{aligned}
& +\frac{1}{2} \sum_{j=1}^{3} \sum_{n=a}^{b} \lambda_{4_{j n}}^{t}\left[\left(\Delta_{j}^{L \dagger} \Delta_{j}^{L}\right) O_{1 n}^{t t}\right]+\sum_{j=1}^{3} \sum_{n \neq l ; n, l=a}^{b} \lambda_{4_{1 n l}^{t}}^{t}\left[\left(\Delta_{j}^{L \dagger} \Delta_{j}^{L}\right) \hat{O}_{1 n}^{t t}+\text { H.c. }\right]+\frac{1}{2} \sum_{n=a}^{b} \lambda_{5 n}^{t}\left[\left\{\Delta_{1}^{L} \mathcal{O}_{1 n}^{t t}\right\}+\text { H.c. }\right] \\
& +\sum_{n, l=a}^{b} \lambda_{5 n l}^{t}\left[\left\{\Delta_{1}^{L} \mathcal{O}_{1 n l}^{t t}\right\}+\text { H.c. }\right]+\frac{1}{2} \sum_{n=a}^{b} \lambda_{6 n}^{t}\left[\left\{\Delta_{2}^{L} \mathcal{O}_{3 n}^{t t}\right\}+\text { H.c. }\right] \\
& +\sum_{n, l=a}^{b} \lambda_{6 n l}^{t}\left[\left\{\Delta_{2}^{L} \mathcal{O}_{3 n l}^{t t}\right\}+\text { H.c. }\right]+\frac{1}{2} \sum_{n=a}^{b} \lambda_{7 n}^{t}\left[\left\{\Delta_{3}^{L} \mathcal{O}_{2 n}^{t t}\right\}+\text { H.c. }\right]+\sum_{n, l=a}^{b} \lambda_{7 n l}^{t}\left[\left\{\Delta_{3}^{L} \mathcal{O}_{2 n l}^{t t}\right\}+\text { H.c. }\right] \\
& +\sum_{n=a}^{b} \sum_{j=1}^{3} \lambda_{8 j n}^{t}\left[\left(\Delta_{j}^{L 2} \tilde{O}_{j n}^{t t}\right)+\text { H.c. }\right]+\sum_{n \neq l ; n, l=a}^{b} \sum_{j=1}^{3} \lambda_{8 j n l}^{t}\left[\left(\Delta_{j}^{L 2} \tilde{O}_{j n l}^{t t}\right)+\text { H.c. }\right] \\
& +\sum_{n=a}^{b}\left[\left\{\lambda_{91 n}^{t}\left(\Delta_{2}^{L} \Delta_{3}^{L} \tilde{O}_{1 n}^{t t}\right)\right\}+\left\{\lambda_{92 n}^{t}\left(\Delta_{1}^{L} \Delta_{3}^{L} \tilde{O}_{2 n}^{t t}\right)\right\}+\left\{\lambda_{93 n}^{t}\left(\Delta_{1}^{L} \Delta_{2}^{L} \tilde{O}_{3 n}^{t t}\right)\right\}+\text { H.c. }\right] \\
& +\sum_{n \neq l ; n, l=a}^{b}\left[\left\{\lambda_{91 n l}^{t}\left(\Delta_{2}^{L} \Delta_{3}^{L} \tilde{O}_{1 n l}^{t t}\right)\right\}+\left\{\lambda_{92 n l}^{t}\left(\Delta_{1}^{L} \Delta_{3}^{L} \tilde{O}_{2 n l}^{t t}\right)\right\}+\left\{\lambda_{93 n l}^{t}\left(\Delta_{1}^{L} \Delta_{2}^{L} \tilde{O}_{3 n l}^{t t}\right)\right\}+\text { H.c. }\right] .
\end{aligned}
$$

\section{Inter-sector terms in the scalar potential}

The terms in the scalar potential involving scalar fields of identical $S U(2)_{L}$ behavior are already taken into account. Apart from them, the scalar potential will also receive contributions from terms generated by combining scalars of two different $S U(2)_{L}$ sectors that constitute the main objective of the following discussion. In this category the combinations of the $S U(2)_{L}$ singlet scalars with that belonging to either of the doublet or the triplet sector. The other variety of inter-sector terms-doublet-triplet typeare not included. This seems to be a reasonable approximation as the vevs of the singlet fields are the largest.

\section{a. Singlet-doublet inter-sector terms}

Let us consider the combinations:

$$
\tilde{T}_{s p}^{s s} \equiv T_{s}\left(\hat{\Delta}_{p}^{R}, \hat{\Delta}_{p}^{R \dagger}\right) ; \quad \tilde{T}_{s p q}^{s s} \equiv T_{s}\left(\hat{\Delta}_{p}^{R}, \hat{\Delta}_{q}^{R \dagger}\right) \quad \text { and } \quad \tilde{T}_{s}^{d d} \equiv T_{s}\left(\Phi, \Phi^{\dagger}\right), \quad(p, q=a, b, c \quad \text { and } \quad p \neq q)
$$

and

$$
\begin{aligned}
O_{1 s p}^{s d} & \equiv O_{1}\left(\tilde{T}_{s}^{d d}, \tilde{T}_{s p}^{s s}\right) ; \quad \hat{O}_{1 s p q}^{s d} \equiv O_{1}\left(\tilde{T}_{s}^{d d}, \tilde{T}_{s p q}^{s s}\right) ; \\
\mathcal{O}_{p \gamma}^{s d} & \equiv O_{\gamma}\left(\hat{\Delta}_{p}^{R}, \tilde{T}_{s}^{d d}\right), \quad(\gamma=1,2,3) \quad \text { and } \quad(p, q=a, b, c \quad \text { with } \quad p \neq q) .
\end{aligned}
$$

Using this notation,

$$
\begin{aligned}
V_{s d}= & \frac{1}{2} \sum_{i=1}^{3}\left[\lambda_{1 i}^{s d}\left(Q_{i}^{s s} Q_{\eta}^{d d}\right)+\left(\lambda_{2 i}^{s d} Q_{i}^{s s} O_{1}^{d d}\right)\right]+\frac{1}{2} \sum_{p=a}^{c} \lambda_{3 p}^{s d}\left[Q_{\eta}^{d d} O_{1 p}^{s s}\right]+\frac{1}{2} \sum_{p \neq q ; p, q=a}^{c}\left[Q_{\eta}^{d d} \hat{O}_{1 p q}^{s s}\right] \\
& +\sum_{p=a}^{c}\left[\lambda_{4 p}^{s d}\left(\left\{\mathcal{O}_{1 p}^{s d}\right\} \Delta_{1}^{R}+\text { H.c. }\right)+\lambda_{5 p}^{s d}\left(\left\{\mathcal{O}_{2 p}^{s d}\right\} \Delta_{2}^{R}+\text { H.c. }\right)+\lambda_{6 p}^{s d}\left(\left\{\mathcal{O}_{3 p}^{s d}\right\} \Delta_{3}^{R}+\text { H.c. }\right)\right] \\
& +\frac{1}{2} \sum_{p=a}^{c} \lambda_{7 p}^{s d}\left[O_{1}^{d d} O_{1 p}^{s s}+\left\{O_{2 p}^{s s}\right\}^{\dagger} O_{2}^{d d}+\left\{O_{2}^{d d}\right\}^{\dagger} O_{2 p}^{s s}+O_{1 s p}^{s d}\right] \\
& +\frac{1}{2} \sum_{p \neq q ; p, q=a}^{c} \lambda_{7 p q}^{s d}\left[O_{1}^{d d} \hat{O}_{1 p q}^{s s}+\left\{\hat{O}_{2 p q}^{s s}\right\}^{\dagger} O_{2}^{d d}+\left\{O_{2}^{d d}\right\}^{\dagger} \hat{O}_{2 p q}^{s s}+\hat{O}_{1 s p q}^{s d}\right] .
\end{aligned}
$$


In the last two terms, a simplifying assumption of using a common couplings $\lambda_{7 p}^{s d}$ and $\lambda_{7 p q}^{s d}$ for the terms in the scalar potential that are generated from various combinations of $\left(\Phi^{\dagger} \Phi\right)\left(\hat{\Delta}^{{ }^{\dagger}} \hat{\Delta}^{R}\right)$, all four of the fields involved being triplets of $A 4$.

\section{b. Singlet-triplet inter-sector terms}

In this case, the following combinations come into play:

$$
\begin{array}{rlrl}
\tilde{T}_{s n}^{t t} & \equiv T_{s}\left(\hat{\Delta}_{n}^{L}, \hat{\Delta}_{n}^{L \dagger}\right) ; & \tilde{T}_{s n l}^{t t} \equiv T_{s}\left(\hat{\Delta}_{n}^{L}, \hat{\Delta}_{l}^{L \dagger}\right) ; \quad O_{1 s n p}^{t s} \equiv O_{1}\left(\tilde{T}_{s n}^{t s}, \tilde{T}_{s p}^{s s}\right) ; \quad \hat{O}_{1 s n p q}^{t s} \equiv O_{1}\left(\tilde{T}_{s n}^{t s}, \tilde{T}_{s p q}^{s s}\right) ; \\
\hat{O}_{1 s n l p}^{t s} \equiv O_{1}\left(\tilde{T}_{s n l}^{t t}, \tilde{T}_{s p}^{s s}\right) ; & & \hat{O}_{1 s n l p q}^{t s} \equiv O_{1}\left(\tilde{T}_{s n l}^{t t}, \tilde{T}_{s p q}^{s s}\right) ; \quad O_{\gamma n p}^{t s} \equiv O_{\gamma}\left(\hat{\Delta}_{p}^{R \dagger}, \hat{\Delta}_{n}^{L}\right) ; & \tilde{O}_{\gamma n p}^{t s} \equiv O_{\gamma}\left(\hat{\Delta}_{p}^{R}, \hat{\Delta}_{n}^{L}\right) ; \\
\mathcal{O}_{\gamma n p}^{t s} \equiv O_{\gamma}\left(\tilde{T}_{s p}^{s s}, \hat{\Delta}_{n}^{L}\right) ; & \tilde{\mathcal{O}}_{\gamma n p}^{t s} \equiv O_{\gamma}\left(\tilde{T}_{s n}^{t s}, \hat{\Delta}_{p}^{R}\right) ; \quad \hat{\mathcal{O}}_{\gamma n p q}^{t s} \equiv O_{\gamma}\left(\tilde{T}_{s p q}^{s s}, \hat{\Delta}_{n}^{L}\right) ; & \tilde{\mathcal{O}}_{\gamma n l p}^{t s} \equiv O_{\gamma}\left(\tilde{T}_{s n l}^{t t}, \hat{\Delta}_{p}^{R}\right),
\end{array}
$$

where $(\gamma=1,2,3) ;(p, q=a, b, c)$ and $(n, l=a, b)$ Needless to mention, $p \neq q$ and $n \neq l$.

Following the convention introduced already,

$$
O_{\gamma n p}^{t s} \equiv O_{\gamma}\left(\hat{\Delta}_{p}^{R \dagger}, \hat{\Delta}_{n}^{L}\right) ; \quad \tilde{O}_{\gamma n p}^{t s} \equiv O_{\gamma}\left(\hat{\Delta}_{p}^{R}, \hat{\Delta}_{n}^{L}\right), \quad(\gamma=1,2,3) ; \quad(p=a, b, c) \quad \text { and } \quad(n=a, b) .
$$

The inter-sector potential for this case is given by

$$
\begin{aligned}
& V_{t s}=\frac{1}{2} \sum_{i=1}^{3} \sum_{j=1}^{3} \lambda_{1 i j}^{t s}\left[Q_{i}^{s s} Q_{j}^{t t}\right]+\frac{1}{2} \sum_{j=1}^{3} \sum_{n=a}^{b} \lambda_{2 j n}^{t s}\left[\left(Q_{j}^{s s} O_{1 n}^{t t}\right)+\text { H.c. }\right]+\frac{1}{2} \sum_{j=1}^{3} \sum_{n \neq l ; n, l=a}^{b} \lambda_{2 j n l}^{t s}\left[\left(Q_{j}^{s s} \hat{O}_{1 n l}^{t t}\right)+\text { H.c. }\right] \\
& +\frac{1}{2} \sum_{i=1}^{3} \sum_{p=a}^{c} \lambda_{3 i p}^{t s}\left[Q_{i}^{t t} O_{1 p}^{s s}\right]+\sum_{i=1}^{3} \sum_{p \neq q ; p, q=a}^{c} \lambda_{3 i p q}^{t s}\left[Q_{i}^{t t} \hat{O}_{1 p q}^{s s}\right]+\frac{1}{2} \sum_{p=a}^{c} \sum_{n=a}^{b} \lambda_{41 n n p p}^{t s}\left[O_{1 n}^{t t} O_{1 p}^{s s}+\left\{O_{2 p}^{s s}\right\}^{\dagger} O_{2 n}^{t t}+\left\{O_{2 n}^{t t}\right\}^{\dagger} O_{2 p}^{s s}+O_{1 s n p}^{t s}\right] \\
& +\frac{1}{2} \sum_{p \neq q ; p, q=a}^{c} \sum_{n=a}^{b} \lambda_{42 n n p q}^{t s}\left[O_{1 n}^{t t} \hat{O}_{1 p q}^{s s}+\left\{\hat{O}_{2 p q}^{s s}\right\}^{\dagger} O_{2 n}^{t t}+\left\{O_{2 n}^{t t}\right\}^{\dagger} \hat{O}_{2 p q}^{s s}+\hat{O}_{1 s n p q}^{t s}\right] \\
& +\frac{1}{2} \sum_{p=a}^{c} \sum_{n \neq l ; n, l=a}^{b} \lambda_{43 n l p p}^{t s}\left[\hat{O}_{1 n l}^{t t} O_{1 p}^{s s}+\left\{O_{2 p}^{s s}\right\}^{\dagger} \hat{O}_{2 n l}^{t t}+\left\{\hat{O}_{2 n l}^{t t}\right\}^{\dagger} O_{2 p}^{s s}+\hat{O}_{1 s n l p}^{t s}\right] \\
& +\frac{1}{2} \sum_{p \neq q ; p, q=a}^{c} \sum_{n \neq l ; n, l=a}^{b} \lambda_{44 n l p q}^{t s}\left[\hat{O}_{1 n l}^{t t} \hat{O}_{1 p q}^{s s}+\left\{\hat{O}_{2 p q}^{s s}\right\}^{\dagger} \hat{O}_{2 n l}^{t t}+\left\{\hat{O}_{2 n l}^{t t}\right\}^{\dagger} \hat{O}_{2 p q}^{s s}+\hat{O}_{1 s n l p q}^{t s}\right] \\
& +\sum_{i=1}^{3} \sum_{p=a}^{c} \sum_{n=a}^{b} \lambda_{5 i p p n}^{t s}\left(\mathcal{O}_{i n p}^{t s} \Delta_{i}^{L \dagger}+\text { H.c. }\right)+\sum_{i=1}^{3} \sum_{p \neq q ; p, q=a}^{c} \sum_{n=a}^{b} \lambda_{5 i p q n}^{t s}\left(\hat{\mathcal{O}}_{i n p q}^{t s} \Delta_{i}^{L \dagger}+\text { H.c. }\right) \\
& +\sum_{i=1}^{3} \sum_{p=a}^{c} \sum_{n=a}^{b} \lambda_{\text {6innp }}^{t s}\left(\tilde{\mathcal{O}}_{i n p}^{t s} \Delta_{i}^{R^{\dagger}}+\text { H.c. }\right)+\sum_{i=1}^{3} \sum_{p=a}^{c} \sum_{n \neq l ; n, l=a}^{b} \lambda_{\text {6inlp }}^{t s}\left(\tilde{\mathcal{O}}_{i n l p}^{t s} \Delta_{i}^{R^{\dagger}}+\text { H.c. }\right) \\
& +\sum_{p=a}^{c} \sum_{n=a}^{b}\left[\lambda_{7}^{t s} O_{1 n p}^{t s}\left(\Delta_{1}^{L^{\dagger}} \Delta_{1}^{R}+\Delta_{2}^{L^{\dagger}} \Delta_{2}^{R}+\Delta_{3}^{L^{\dagger}} \Delta_{3}^{R}\right)+\text { H.c. }\right]+\sum_{p=a}^{c} \sum_{n=a}^{b}\left[\lambda_{8}^{t s} O_{2 n p}^{t s}\left(\Delta_{1}^{L \dagger} \Delta_{3}^{R}+\Delta_{2}^{L^{\dagger} \dagger} \Delta_{1}^{R}+\Delta_{3}^{L^{\dagger}} \Delta_{2}^{R}\right)+\text { H.c. }\right] \\
& +\sum_{p=a}^{c} \sum_{n=a}^{b}\left[\lambda_{9}^{t s} O_{3 n p}^{t s}\left(\Delta_{3}^{L \dagger} \Delta_{1}^{R}+\Delta_{1}^{L \dagger} \Delta_{2}^{R}+\Delta_{2}^{L \dagger} \Delta_{3}^{R}\right)+\text { H.c. }\right]+\sum_{p=a}^{c} \sum_{n=a}^{b}\left[\lambda_{10}^{t s} \tilde{O}_{3 n p}^{t s}\left(\Delta_{3}^{L \dagger} \Delta_{1}^{R \dagger}+\Delta_{1}^{L \dagger} \Delta_{3}^{R^{\dagger}}+\Delta_{2}^{L^{\dagger}} \Delta_{2}^{R \dagger}\right)+\text { H.c. }\right] \\
& +\sum_{p=a}^{c} \sum_{n=a}^{b}\left[\lambda_{11}^{t s} \tilde{O}_{2 n p}^{t s}\left(\Delta_{2}^{L \dagger} \Delta_{1}^{R \dagger}+\Delta_{1}^{L \dagger} \Delta_{2}^{R \dagger}+\Delta_{3}^{L \dagger} \Delta_{3}^{R \dagger}\right)+\text { H.c. }\right]+\sum_{p=a}^{c} \sum_{n=a}^{b}\left[\lambda_{12}^{t s} \tilde{O}_{1 n p}^{t s}\left(\Delta_{1}^{L \dagger} \Delta_{1}^{R \dagger}+\Delta_{3}^{L \dagger} \Delta_{2}^{R \dagger}+\Delta_{2}^{L \dagger} \Delta_{3}^{R \dagger}\right)+\text { H.c. }\right] \text {. }
\end{aligned}
$$

It must be noted that while writing the last $\lambda_{7-12}^{t s}$ terms the different couplings corresponding to the combinations of $O_{i n p}^{t s}$ with $\left(\Delta_{i}^{L \dagger} \Delta_{j}^{R}\right)$ and $\tilde{O}_{i n p}^{t s}$ with $\left(\Delta_{i}^{L^{\dagger}} \Delta_{j}^{R \dagger}\right)$ are set to be equal. 


\section{The conditions for minimization}

With the scalar potential in hand, it is necessary to derive the conditions for which the particular vev configurations used in this model—see Eqs. (3)-(5) and Table III-corresponds to the local minimum. For immediate reference the vevs are

$$
\begin{gathered}
\left\langle\Phi^{0}\right\rangle=\frac{v}{\sqrt{3}}\left(\begin{array}{l}
1 \\
1 \\
1
\end{array}\right), \quad\left\langle\eta^{0}\right\rangle=u, \quad\left\langle\hat{\Delta}_{a}^{L 0}\right\rangle=v_{L a}\left(\begin{array}{l}
1 \\
0 \\
0
\end{array}\right), \quad\left\langle\hat{\Delta}_{b}^{L 0}\right\rangle=v_{L b}\left(\begin{array}{l}
1 \\
1 \\
1
\end{array}\right), \quad\left\langle\Delta_{1}^{L 0}\right\rangle=\left\langle\Delta_{2}^{L 0}\right\rangle=\left\langle\Delta_{3}^{L 0}\right\rangle=u_{L}, \\
\left\langle\hat{\Delta}_{a}^{R 0}\right\rangle=v_{R a}\left(\begin{array}{l}
1 \\
1 \\
1
\end{array}\right), \quad\left\langle\hat{\Delta}_{b}^{R 0}\right\rangle=v_{R b}\left(\begin{array}{c}
1 \\
\omega \\
\omega^{2}
\end{array}\right), \quad\left\langle\hat{\Delta}_{c}^{R 0}\right\rangle=v_{R c}\left(\begin{array}{c}
1 \\
\omega^{2} \\
\omega
\end{array}\right), \\
\left\langle\Delta_{1}^{R 0}\right\rangle=u_{1 R}, \quad\left\langle\Delta_{2}^{R 0}\right\rangle=u_{2 R}, \quad\left\langle\Delta_{3}^{R 0}\right\rangle=u_{3 R} .
\end{gathered}
$$

where the $S U(2)_{L}$ nature of the scalars has been suppressed.

Eq. (B26) shows that the $A 4$ triplet fields $-\hat{\Delta}^{L, R}$ and $\Phi-$ have vev configurations that have been verified to be the global minima in [25]. This result was for a single $A 4$ triplet considered in isolation. In the current scenario since many other fields are involved, it is not straightforward to directly adopt the conclusions of [25].

The conditions for which the vev configurations shown in Eqs. (3)-(5) correspond to minimum are shown sector by sector.

For minima of the scalar potential, the first derivatives of the scalar potential with respect to the vevs have to vanish and the second derivatives have to satisfy some conditions. Since the scalar sector is very rich, the expressions look very complicated. The conditions arising by setting the first derivatives to be zero have been discussed for each of the $S U(2)_{L}$ sectors. As a sample, constraints coming from the second derivatives have been shown only for the $S U(2)_{L}$ singlet sector. Similar exercise can be carried out for the other $S U(2)_{L}$ sectors but are not presented here.

\section{a. $S U(2)_{L}$ singlet sector}

The $S U(2)_{L}$ singlet vevs are much larger than those of the doublet and triplet scalars. Thus, it is safe to neglect the contributions to the minimization equations from the intersector terms.

Let us remind ourselves that $v_{R p}(p=a, b, c)$ are real and define

$\tilde{v}_{R a_{1}} \equiv v_{R a}, \quad \tilde{v}_{R a_{2}} \equiv v_{R a}, \quad \tilde{v}_{R a_{3}} \equiv v_{R a} ;$

$\tilde{v}_{R b_{1}} \equiv v_{R b}, \quad \tilde{v}_{R b_{2}} \equiv v_{R b} \omega, \quad \tilde{v}_{R b_{3}} \equiv v_{R a} \omega^{2} ;$

$\tilde{v}_{R c_{1}} \equiv v_{R c}, \quad \tilde{v}_{R c_{2}} \equiv v_{R c} \omega^{2}, \quad \tilde{v}_{R c_{3}} \equiv v_{R c} \omega$.

For ease of presentation, let us set the following masses and couplings equal:

$$
\begin{aligned}
m_{\Delta_{1}^{R}}^{2} & =m_{\Delta_{2}^{R}}^{2}=m_{\Delta_{3}^{R}}^{2}=m_{R 1}^{2} ; \quad m_{\hat{\Delta}_{a}^{R}}^{2}=m_{\hat{\Delta}_{b}^{R}}^{2}=m_{\hat{\Delta}_{c}^{R}}^{2}=m_{R 2}^{2} ; \quad m_{a b}^{2}=m_{a c}^{2}=m_{b c}^{2}=m_{R 3}^{2} ; \\
\lambda_{1 i}^{s} & =\lambda_{1}^{s} \quad \forall(i=1,2,3) ; \quad \lambda_{221}^{s}=\lambda_{231}^{s}=\lambda_{223}^{s}=\lambda_{2}^{s} ; \quad \lambda_{3 a}^{s}=\lambda_{3 b}^{s}=\lambda_{3 c}^{s}=\lambda_{3}^{s} ; \quad \tilde{\lambda}_{3 a}^{s}=\tilde{\lambda}_{3 b}^{s}=\tilde{\lambda}_{3 c}^{s}=\tilde{\lambda}_{3}^{s} ; \\
\lambda_{3 a b}^{s} & =\lambda_{3 a c}^{s}=\lambda_{3 b c}^{s}=\hat{\lambda}_{3}^{s} ; \quad \lambda_{4 i p}^{s}=\lambda_{4}^{s} \quad \forall(p=a, b, c) \quad \text { and } \quad(i=1,2,3) ; \\
\lambda_{41 a b}^{s} & =\lambda_{41 a c}^{s}=\lambda_{41 b c}^{s}=\lambda_{42 a b}^{s}=\lambda_{42 a c}^{s}=\lambda_{42 b c}^{s}=\lambda_{43 a b}^{s}=\lambda_{43 a c}^{s}=\lambda_{43 b c}^{s}=\tilde{\lambda}_{4}^{s} ; \\
\lambda_{5 a}^{s} & =\lambda_{5 b}^{s}=\lambda_{5 c}^{s}=\lambda_{5}^{s} ; \quad \lambda_{5 a b}^{s}=\lambda_{5 a c}^{s}=\lambda_{5 b c}^{s}=\tilde{\lambda}_{5}^{s} ; \quad \lambda_{6 a}^{s}=\lambda_{6 b}^{s}=\lambda_{6 c}^{s}=\lambda_{6}^{s} ; \quad \lambda_{6 a b}^{s}=\lambda_{6 a c}^{s}=\lambda_{6 b c}^{s}=\tilde{\lambda}_{6}^{s} ; \\
\lambda_{7 a}^{s} & =\lambda_{7 b}^{s}=\lambda_{7 c}^{s}=\lambda_{7}^{s} ; \quad \lambda_{7 a b}^{s}=\lambda_{7 a c}^{s}=\lambda_{7 b c}^{s}=\tilde{\lambda}_{7}^{s} ; \quad \lambda_{8 i p}^{s}=\lambda_{8}^{s} \quad \forall(p=a, b, c) \quad \text { and } \quad(i=1,2,3) ; \\
\lambda_{81 a b}^{s} & =\lambda_{81 a c}^{s}=\lambda_{81 b c}^{s}=\lambda_{82 a b}^{s}=\lambda_{82 a c}^{s}=\lambda_{82 b c}^{s}=\lambda_{83 a b}^{s}=\lambda_{83 a c}^{s}=\lambda_{83 b c}^{s}=\tilde{\lambda}_{8}^{s} ; \\
\lambda_{9 i p}^{s} & =\lambda_{9}^{s} \quad \forall(p=a, b, c) \quad \text { and } \quad(i=1,2,3) ; \\
\lambda_{91 a b}^{s} & =\lambda_{91 a c}^{s}=\lambda_{91 b c}^{s}=\lambda_{92 a b}^{s}=\lambda_{92 a c}^{s}=\lambda_{92 b c}^{s}=\lambda_{93 a b}^{s}=\lambda_{93 a c}^{s}=\lambda_{93 b c}^{s}=\tilde{\lambda}_{9}^{s} .
\end{aligned}
$$

With the help of the singlet sector potential in Eq. (B10), the equalities in Eq. (B30) and the vev in Eqs. (3)-(5) one can obtain 


$$
\begin{aligned}
\frac{\left.\partial V_{\text {singlet }}\right|_{\text {min }}}{\partial u_{1 R}^{*}}=0 & \\
\Rightarrow & m_{R 1}^{2} u_{1 R}+\lambda_{1}^{s}\left(u_{1 R}^{*} u_{1 R}^{2}\right)+\lambda_{2}^{s}\left[\left(u_{2 R}^{*} u_{2 R}\right)+\left(u_{3 R}^{*} u_{3 R}\right)\right]+\frac{3 \lambda_{4}^{s}}{2} u_{1 R}\left[v_{R a}^{2}+v_{R b}^{2}+v_{R c}^{2}\right]+3 \lambda_{5}^{s} v_{R a}^{3} \\
& -3 \tilde{\lambda}_{5}^{s} v_{R a}\left(v_{R b}^{2}+v_{R c}^{2}\right)+6 \lambda_{8}^{s} u_{1 R}^{*} v_{R a}^{2}+6 \tilde{\lambda}_{8}^{s} u_{1 R}^{*} v_{R b} v_{R c} \\
+ & 3 \lambda_{9}^{s}\left(u_{3 R}^{*} v_{R b}^{2}+u_{2 R}^{*} v_{R c}^{2}\right)+3 \tilde{\lambda}_{9}^{s}\left[v_{R a}\left(u_{2 R}^{*} v_{R b}+u_{3 R}^{*} v_{R c}\right)\right]=0 \\
\frac{\left.\partial V_{\text {singlet }}\right|_{\text {min }}=}{\partial \tilde{v}_{R a_{1}}^{*}=} & \frac{\partial V_{\text {singlet }\left.\right|_{\text {min }}}=0}{\partial v_{R a_{1}}^{*}} \\
\Rightarrow & m_{R 2}^{2} v_{R a}+m_{R 3}^{2}\left(v_{R b}+v_{R c}\right)+\frac{7}{2} \lambda_{3}^{s} v_{R a}^{3}+\left(3 \hat{\lambda}_{3}+\frac{\tilde{\lambda}_{3}^{s}}{8}\right) v_{R a}\left(v_{R b}^{2}+v_{R c}^{2}\right) \\
& +\left[\frac{\lambda_{4}^{s}}{2} v_{R a}+\tilde{\lambda}_{4}^{s}\left(v_{R b}+v_{R c}\right)\right]\left(u_{1 R}^{*} u_{1 R}+u_{2 R}^{*} u_{2 R}+u_{3 R}^{*} u_{3 R}\right)+\lambda_{5}^{s} v_{R a}^{2}\left(u_{1 R}^{*}+2 u_{1 R}\right) \\
& -\tilde{\lambda}_{5}^{s}\left[\left(v_{R a} v_{R b}+v_{R a} v_{R c}\right)\left(2 u_{1 R}+u_{1 R}^{*}\right)\right]+\lambda_{6}^{s} v_{R a}^{2}\left(u_{2 R}^{*}-u_{2 R}\right) \\
& +\tilde{\lambda}_{6}^{s}\left[u_{2 R}\left(2 v_{R b}^{2}-v_{R c}^{2}-v_{R a} v_{R b}+2 v_{R b} v_{R c}\right)-u_{2 R}^{*} v_{R a}\left(v_{R b}+v_{R c}\right)\right]+\lambda_{7}^{s} v_{R a}^{2}\left(u_{3 R}^{*}-u_{3 R}\right) \\
& +\tilde{\lambda}_{7}^{s}\left[u_{3 R}\left(2 v_{R a} v_{R b}-v_{R a} v_{R c}-v_{R b}^{2}+2 v_{R c}^{2}\right)-u_{3 R}^{*} v_{R a}\left(v_{R b}+v_{R c}\right)\right] \\
& +\left(u_{1 R}^{2}+u_{2 R}^{2}+u_{3 R}^{2}\right)\left[2 \lambda_{8}^{s} v_{R a}+\tilde{\lambda}_{8}^{s}\left(v_{R b}+v_{R c}\right)\right] \\
& +\left(u_{1 R}^{*} u_{2 R}+u_{1 R}^{*} u_{3 R}+u_{2 R}^{*} u_{3 R}\right)\left[2 \lambda_{9}^{s} v_{R a}+\tilde{\lambda}_{9}^{s}\left(v_{R b}+v_{R c}\right)\right]=0 .
\end{aligned}
$$

Besides the first derivatives discussed above, second derivatives are also needed to established minimality. For example,

$$
\frac{\left.\partial^{2} V_{\text {singlet }}\right|_{\min }}{\partial u_{1 R}^{* 2}}>0 \Rightarrow \lambda_{1}^{s} u_{1 R}^{2}+6 \lambda_{8}^{s} v_{R a}^{2}+6 \tilde{\lambda}_{8}^{s} v_{R b} v_{R c}>0
$$

and

$$
\frac{\left.\partial^{2} V_{\text {singlet }}\right|_{\text {min }}}{\partial \tilde{v}_{R a_{1}}^{* 2}}>0 \Rightarrow \lambda_{3}^{s} v_{R a}^{2}+4 \hat{\lambda}_{3}\left(v_{R b}^{2}+v_{R c}^{2}\right)+2 \lambda_{8}^{s}\left(u_{1 R}^{2}+u_{2 R}^{2}+u_{3 R}^{2}\right)+2 \lambda_{8}^{s}\left(u_{2 R} u_{3 R}+u_{1 R} u_{2 R}+u_{1 R} u_{3 R}\right)>0
$$

Further mixed derivatives such as

$$
\frac{\left.\partial^{2} V_{\text {singlet }}\right|_{\min }}{\partial u_{1 R}^{*} \tilde{v}_{R a_{1}}}=\frac{\lambda_{4}^{s}}{2} v_{R a} u_{1 R}+\tilde{\lambda}_{4}^{s}\left(v_{R b}+v_{R c}\right) u_{1 R}+\lambda_{5}^{s} v_{R a}^{2}-\tilde{\lambda}_{5}^{s} v_{R a}\left(v_{R b}+v_{R c}\right)
$$

are also necessary to establish minimality in the most general case. The results presented for the first and second derivatives are calculated using the most general expression of the scalar potential in terms of the vevs and putting $\left(v_{R a 1}=v_{R a 2}=v_{R a 3}=v_{R a}\right), \quad\left(v_{R b 1}=v_{R b}, v_{R b 2}=\omega v_{R b}\right.$, $\left.v_{R b 3}=\omega^{2} v_{R b}\right)$ and $\left(v_{R c 1}=v_{R c}, v_{R c 2}=\omega^{2} v_{R c}, v_{R c 3}=\right.$ $\left.\omega v_{R c}\right)$ where $v_{R a}, v_{R b}, v_{R c}$ are real. Needless to mention that $\tilde{v}_{R p_{i}}^{*}=\tilde{v}_{R p_{i}}$ for $(p=a, i=1,2, \quad 3)$ and ( $p=b, c \quad$ and $\quad i=1$ ). Similar equations can be obtained by minimizing the potential with respect to $u_{2 R}, u_{3 R}$, $\tilde{v}_{R a 2}, \tilde{v}_{R a 3}$ and $\tilde{v}_{R p i}$ for $(p=b, c)$ and $(i=1,2,3)$. For the sake of brevity those are not mentioned. Similar strategy will be adopted for the $S U(2)_{L}$ doublet and $S U(2)_{L}$ triplet sector. It is worth noting that this exercise for all the three sectors are for illustrative purpose only and the minimization equations are achieved by setting the different couplings equal.

\section{b. $S U(2)_{L}$ doublet sector}

For this sector contributions from both the doublet sector itself-Eq. (B13) - together with the singlet-doublet intersector are considered. Let us define $V_{\mathcal{D}}=V_{\text {doublet }}+V_{s d}$. Also let us call $\left\langle\Phi_{i}\right\rangle \equiv v_{i}$ where $v_{1}=v_{2}=v_{3}=\frac{v}{\sqrt{3}}, v$ being real. 
The following couplings are set to be equal:

$$
\begin{aligned}
\lambda_{1 i}^{s d}=\lambda_{1}^{s d}, & \lambda_{2 i}^{s d}=\lambda_{2}^{s d} \quad \forall(i=1,2,3) ; \\
\lambda_{3 p}^{s d}=\lambda_{3}^{s d}, \quad \lambda_{4 p}^{s d}=\lambda_{4}^{s d}, & \lambda_{5 p}^{s d}=\lambda_{5}^{s d}, \quad \lambda_{6 p}^{s d}=\lambda_{6}^{s d}, \lambda_{7 p}^{s d}=\lambda_{7}^{s d} \quad \forall(p=a, b, c) ; \\
\lambda_{3 a b}^{s d}=\lambda_{3 a c}^{s d}=\lambda_{3 b c}^{s d}=\tilde{\lambda}_{3}^{s d} ; & \lambda_{7 a b}^{s d}=\lambda_{7 a c}^{s d}=\lambda_{7 b c}^{s d}=\tilde{\lambda}_{7}^{s d} .
\end{aligned}
$$

For the vevs in Eqs. (B26)-(B28) correspond to the minimum of the scalar potential it is necessary to satisfy the following conditions:

$$
\frac{\left.\partial V_{\mathcal{D}}\right|_{\min }}{\partial u^{*}}=0 \Rightarrow u\left[m_{\eta}^{2}+\lambda_{1}^{d} u^{*} u+\lambda_{3}^{d} v^{2}+\lambda_{1}^{s d} \sum_{i=1}^{3}\left(u_{i R}^{*} u_{i R}\right)+\frac{3}{2} \lambda_{3}^{s d} \sum_{p=a}^{c} v_{R p}^{2}\right]=0 .
$$

and

$$
\begin{aligned}
\frac{\left.\partial V_{\mathcal{D}}\right|_{\min }}{\partial v_{1}^{*}}= & 0 \\
\Rightarrow & \frac{v}{\sqrt{3}}\left[m_{\Phi}^{2}+2 \lambda_{2}^{d} \frac{v^{2}}{3}+\frac{\lambda_{3}^{d}}{2}\left(u^{*} u\right)+\frac{\lambda_{2}^{s d}}{2} \sum_{i=1}^{3}\left(u_{i R}^{*} u_{i R}\right)+\frac{\lambda_{4}^{s d}}{2}\left(2 v_{R a}-v_{R b}-v_{R c}\right)\left(u_{1 R}+u_{1 R}^{*}\right)\right. \\
& +\frac{\lambda_{7}^{s d}}{2}\left[\left(\sum_{p=a}^{c} 3 v_{R p}^{2}\right)+\frac{1}{2}\left(2 v_{R a}^{2}-v_{R b}^{2}-v_{R c}^{2}\right)\right] \\
& \left.+\frac{\tilde{\lambda}_{7}^{s d}}{2}\left[6\left(v_{R a} v_{R b}+v_{R a} v_{R c}+v_{R b} v_{R c}\right)+\frac{1}{2}\left(v_{R a} v_{R b}+v_{R a} v_{R c}-2 v_{R b} v_{R c}\right)\right]\right]=0 .
\end{aligned}
$$

In order to satisfy Eqs. (B37) and (B38), some degree of fine-tuning is necessary that involve both $S U(2)_{L}$ doublet and singlet vev of varying magnitudes. Similar equations can be obtained by minimizing the potential with respect to $v_{2}^{*}$ and $v_{3}^{*}$.

\section{c. $S U(2)_{L}$ triplet sector}

In analogy to the doublet sector, let us define $V_{\mathcal{T}}=V_{\text {triplet }}+V_{t s}$ using Eqs. (B19) and (B25). Let us also recall, $v_{L a 1}=v_{L a}, v_{L a 2}=v_{L a 3}=0$ and $v_{L b 1}=v_{L b 2}=v_{L b 3}=v_{L b}$.

This sector has several couplings involved. For simplicity of presentation, let us implement the following choices:

$$
\begin{aligned}
m_{\Delta_{1}^{L}} & =m_{\Delta_{2}^{L}}=m_{\Delta_{3}^{L}}=m_{t 1} ; \quad m_{\hat{\Delta}_{a}^{L}}=m_{\hat{\Delta}_{b}^{L}}=m_{t 2} ; \quad m_{a b}=m_{t 3} ; \quad \lambda_{1 i}^{t}=\lambda_{1}^{t}, \quad \forall(i=1,2,3) ; \\
\lambda_{221}^{t} & =\lambda_{232}^{t}=\lambda_{231}^{t}=\lambda_{2}^{t} ; \quad \lambda_{3 a}^{t}=\lambda_{3 b}^{t}=\lambda_{3}^{t} ; \lambda_{3 a b}^{t}=\hat{\lambda}_{3}^{t} ; \quad \tilde{\lambda}_{3 a b}^{t}=\tilde{\lambda}_{3}^{t} ; \\
\lambda_{4 j n} & =\lambda_{4 j n l}=\lambda_{4} ; \quad \lambda_{8 j n}=\lambda_{8 j n l}=\lambda_{8} ; \quad \lambda_{9 j n}=\lambda_{9 j n l}=\lambda_{9}, \quad \forall(j=1,2,3), \quad(n, l=a, b) \quad \text { and } \quad n \neq l ; \\
\lambda_{5 a}^{t} & =\lambda_{5 b}^{t}=\lambda_{5 a b}^{t}=\lambda_{5}^{t} ; \quad \lambda_{6 a}^{t}=\lambda_{6 b}^{t}=\lambda_{6 a b}^{t}=\lambda_{6}^{t} ; \quad \lambda_{7 a}^{t}=\lambda_{7 b}^{t}=\lambda_{7 a b}^{t}=\lambda_{7}^{t} ; \\
\lambda_{1 i j}^{t s} & =\lambda_{1}^{t s}, \quad \forall(i, j=1,2,3) \quad \text { and } i \neq j ; \quad \lambda_{2 j n}^{t s}=\lambda_{2 j n l}^{t s}, \quad \forall(j=1,2,3), \quad(n, l=a, b) \quad \text { and } \quad n \neq l ; \\
\lambda_{3 p i}^{t s} & =\lambda_{3 p q i}^{t s}, \quad \forall(i=1,2,3), \quad(p, q=a, b, c) \quad \text { and } \quad p \neq q ; \\
\lambda_{4 j n n p p}^{t s} & =\lambda_{4 j n l p p}^{t s}=\lambda_{4 j n n p q}^{t s}=\lambda_{4 j n l p q}^{t s}=\lambda_{4}^{t s}, \quad \forall(j=1,2,3), \quad(p, q=a, b, c), \quad(n, l=a, b) \quad \text { and } \quad p \neq q, \quad n \neq l ; \\
\lambda_{5 j p p n}^{t s} & =\lambda_{5 j p q n}^{t s}=\lambda_{5}^{t s}, \quad \forall(j=1,2,3), \quad(p=a, b, c), \quad(n=a, b) ; \\
\lambda_{6 j n n p}^{t s} & =\lambda_{6 j n l p}^{t s}=\lambda_{6}^{t s}, \quad \forall(j=1,2,3), \quad(p=a, b, c), \quad(n, l=a, b) \quad \text { and } \quad n \neq l .
\end{aligned}
$$

In order to minimize $V_{\mathcal{T}}$ such that one can arrive at the vevs furnished in Eqs. (B26)-(B28), the following conditions are to be ensured: 


$$
\begin{aligned}
\frac{\left.\partial V_{\mathscr{T}}\right|_{\min }}{\partial u_{L 1}^{*}} & =0 \\
& \Rightarrow u_{L}\left[m_{t 1}^{2}+\left(u_{L}^{*} u_{L}\right)\left(\lambda_{1}^{t}+\lambda_{2}^{t}\right)+\frac{\lambda_{4}^{t}}{2}\left(v_{L a}^{2}+3 v_{L b}^{2}+2 v_{L a} v_{L b}\right)\right]+\lambda_{5}^{t} v_{L b}^{2}\left(3 v_{L b}+v_{L a}\right) \\
& +2 \lambda_{8}^{t} u_{L}^{*}\left(v_{L a}^{2}+3 v_{L b}^{2}+v_{L a} v_{L b}\right)+2 \lambda_{9}^{t} v_{L a}\left(v_{L a}+v_{L b}\right)+\lambda_{1}^{t s} u_{L} \sum_{i=1}^{3}\left(u_{i R}^{*} u_{i R}\right) \\
& +\frac{3}{2} \lambda_{3}^{t s} u_{L}\left[v_{R a}^{2}+v_{R b}^{2}+v_{R c}^{2}+v_{R b} v_{R c}\right] \\
& +\lambda_{5}^{t s}\left(v_{L a}+3 v_{L b}\right)\left[2 v_{R a}^{2}-v_{R b}^{2}-v_{R c}^{2}-2 v_{R a}\left(v_{R a}+v_{R b}\right)+4 v_{R b} v_{R c}\right] \\
& +\left[\left(\lambda_{7}^{t s} u_{1 R}+\lambda_{12}^{t s} u_{1 R}^{*}\right)\left[v_{L a}\left(v_{R a}+v_{R b}+v_{R c}\right)+3 v_{R a} v_{L b}\right]\right] \\
& +\left[\left(\lambda_{8}^{t s} u_{3 R}+\lambda_{10}^{t s} u_{3 R}^{*}\right)\left[v_{L a}\left(v_{R a}+v_{R b}+v_{R c}\right)+3 v_{R c} v_{L b}\right]\right] \\
& +\left[\left(\lambda_{9}^{t s} u_{2 R}+\lambda_{11}^{t s} u_{2 R}^{*}\right)\left[v_{L a}\left(v_{R a}+v_{R b}+v_{R c}\right)+3 v_{R b} v_{L b}\right]\right]=0 .
\end{aligned}
$$

Also, one gets

$$
\begin{aligned}
\frac{\left.\partial V_{\mathcal{T}}\right|_{\min }}{\partial v_{L a_{1}}^{*}}= & 0 \\
\Rightarrow & v_{L a}\left[m_{t 1}^{2}+m_{t 2}^{2}+2 \lambda_{3}^{t} v_{L a}^{2}+4 \hat{\lambda}_{3}^{t} v_{L b}^{2}+\frac{3}{2} \tilde{\lambda}_{3}^{t} v_{L b}^{2}\right]+\frac{3}{2} \lambda_{4}^{t}\left(u_{L}^{*} u_{L}\right)\left(v_{L a}+v_{L b}\right) \\
& +\left(2 \lambda_{5}^{t}-\lambda_{6}^{t}-\lambda_{7}^{t}\right) v_{L b}^{2} u_{L}+\lambda_{8}^{t} u_{L}^{2}\left(2 v_{L a}+3 v_{L b}\right)+\lambda_{9}^{t} u_{L}^{2}\left(2 v_{L a}+v_{L b}\right) \\
& +\frac{\lambda_{2}^{t s}}{2}\left[\left(v_{L a}+v_{L b}\right) \sum_{i=1}^{3} u_{i R}^{*} u_{i R}\right]+\frac{\lambda_{4}^{t s}}{2}\left[3\left(v_{L a}+v_{L b}\right) \sum_{p=a}^{c} v_{R p}^{2}+\frac{1}{2} v_{L b}\left(2 v_{R a}^{2}-v_{R b}^{2}-v_{R c}^{2}\right)\right] \\
& +\tilde{\lambda}_{4}^{t s}\left[3\left(v_{L a}+v_{L b}\right)\left(v_{R a} v_{R b}+v_{R b} v_{R c}\right)+\frac{1}{2} v_{L b}\left(v_{R a} v_{R b}+v_{R a} v_{R c}+v_{R b} v_{R c}\right)\right] \\
& +\lambda_{5}^{t s}\left[3 u_{L}\left(2 v_{R a}^{2}-v_{R b}^{2}-v_{R c}^{2}-2 v_{R a} v_{R b}-2 v_{R a} v_{R c}+4 v_{R b} v_{R c}\right)\right] \\
& +\left[u_{L}\left(v_{R a}+v_{R b}+v_{R c}\right)\right]\left[\left(u_{1 R}^{*}+u_{2 R}^{*}+u_{3 R}^{*}\right)\left(\lambda_{7}^{t s}+\lambda_{8}^{t s}+\lambda_{9}^{t s}\right)\right] \\
& \left.+\left[\left(u_{1 R}+u_{2 R}+u_{3 R}\right)\left(\lambda_{10}^{t s}+\lambda_{11}^{t s}+\lambda_{12}^{t s}\right)\right]\right]=0 .
\end{aligned}
$$

It is worth noticing that certain fine-tuning is essential to satisfy Eqs. (B40)-(B41). Also similar equations can be obtained by minimizing the potential with respect to $u_{L j}^{*}$, where $(j=2,3), v_{L n i}^{*}$ where for $n=b$ one has $(i=1,2,3)$ and for $n=a$ we have $(i=2,3)$. Those are not mentioned here. This exercise is performed to illustrate the scenario in a simplified limit achieved by setting several masses and couplings to be equal.

\section{APPENDIX C: FLAVOR BASIS FORM OF THE MASS MATRICES}

Mass matrices expressed in the Lagrangian basis in Eqs. (6) and (7) can be transformed to simpler forms in the flavor basis as in Eqs. (9) and (10) with the help of a unitary transformation written in Eq. (8). Certain straightforward algebraic calculations related to this derivation of the forms the mass matrices in the flavor basis is furnished in this Appendix.

The Lagrangian in Eq. (2) produces the following mass matrix for the charged leptons and the left-handed Majorana neutrinos,

$$
M_{e \mu \tau}=\frac{v}{\sqrt{3}}\left(\begin{array}{ccc}
y_{1} & y_{2} & y_{3} \\
y_{1} & \omega y_{2} & \omega^{2} y_{3} \\
y_{1} & \omega^{2} y_{2} & \omega y_{3}
\end{array}\right), \quad M_{\nu L}=\left(\begin{array}{ccc}
\left(Y_{1}^{L}+2 Y_{2}^{L}\right) u_{L} & \frac{1}{2} \hat{Y}_{b}^{L} v_{L b} & \frac{1}{2} \hat{Y}_{b}^{L} v_{L b} \\
\frac{1}{2} \hat{Y}_{b}^{L} v_{L b} & \left(Y_{1}^{L}-Y_{2}^{L}\right) u_{L} & \frac{1}{2}\left(\hat{Y}_{a}^{L} v_{L a}+\hat{Y}_{b}^{L} v_{L b}\right) \\
\frac{1}{2} \hat{Y}_{b}^{L} v_{L b} & \frac{1}{2}\left(\hat{Y}_{a}^{L} v_{L a}+\hat{Y}_{b}^{L} v_{L b}\right) & \left(Y_{1}^{L}-Y_{2}^{L}\right) u_{L}
\end{array}\right),
$$


where the Yukawa coupling $Y_{2}^{L}$ is chosen to be equal to $Y_{3}^{L}$. Also, $y_{1} v=m_{e}, y_{2} v=m_{\mu}, y_{3} v=m_{\tau}$ is satisfied. The dominant type-II seesaw component of the neutrino mass matrix, $M_{\nu L}$, gives rise to the atmospheric splitting and maximal atmospheric mixing but is devoid of solar splitting and is therefore characterized by two masses $m_{1}^{(0)}$ and $m_{3}^{(0)}$. It is useful to define $m^{ \pm} \equiv m_{3}^{(0)} \pm m_{1}^{(0)}$. Thus, $m^{-}$is positive (negative) for normal (inverted) ordering. Certain identifications of the vev and Yukawa products are essential viz. $3\left(Y_{1}^{L}+2 Y_{2}^{L}\right) u_{L}=\left(m_{3}^{(0)}+m^{+}\right), 6\left(Y_{1}^{L}-\right.$ $\left.Y_{2}^{L}\right) u_{L}=\hat{Y}_{a}^{L} v_{L a}=m^{+}$and $3 \hat{Y}_{b}^{L} v_{L b}=-2 m^{-}$to generate the desired structures of the mass matrices as presented in Eq. (9). The neutrino Dirac mass matrix and the righthanded Majorana neutrino mass matrix in the Lagrangian basis are

$$
M_{D}=f u \mathbb{I}, \quad M_{\nu R}=m_{R}\left(\begin{array}{ccc}
\chi_{1} & \chi_{6} & \chi_{5} \\
\chi_{6} & \chi_{2} & \chi_{4} \\
\chi_{5} & \chi_{4} & \chi_{3}
\end{array}\right)
$$

where

$$
\begin{aligned}
m_{R} \chi_{1} & \equiv\left(Y_{1}^{R} u_{1 R}+Y_{2}^{R} u_{2 R}+Y_{3}^{R} u_{3 R}\right) \\
m_{R} \chi_{2} & \equiv\left(Y_{1}^{R} u_{1 R}+\omega Y_{2}^{R} u_{2 R}+\omega^{2} Y_{3}^{R} u_{3 R}\right) \\
m_{R} \chi_{3} & \equiv\left(Y_{1}^{R} u_{1 R}+\omega^{2} Y_{2}^{R} u_{2 R}+\omega Y_{3}^{R} u_{3 R}\right) \\
m_{R} \chi_{4} & \equiv \frac{1}{2}\left(\hat{Y}_{a}^{R} v_{R a}+\hat{Y}_{b}^{R} v_{R b}+\hat{Y}_{c}^{R} v_{R c}\right) \\
m_{R} \chi_{5} & \equiv \frac{1}{2}\left(\hat{Y}_{a}^{R} v_{R a}+\omega \hat{Y}_{b}^{R} v_{R b}+\omega^{2} \hat{Y}_{c}^{R} v_{R c}\right) \\
m_{R} \chi_{6} & \equiv \frac{1}{2}\left(\hat{Y}_{a}^{R} v_{R a}+\omega^{2} \hat{Y}_{b}^{R} v_{R b}+\omega \hat{Y}_{c}^{R} v_{R c}\right) .
\end{aligned}
$$

Here $m_{R}$ is the right-handed Majorana neutrino mass scale and $\chi_{i}$ are dimensionless $\mathcal{O}(1)$ quantities. In order to achieve the right-handed Majorana neutrino mass matrix of the form expressed in Eq. (10), the vev and Yukawa couplings products have to obey

$$
\begin{aligned}
& Y_{1}^{R} u_{1 R}=m_{R}\left(r_{11}+2 r_{23}\right), \quad Y_{2}^{R} u_{2 R}=m_{R}\left(r_{22}+2 r_{13}\right), \\
& Y_{3}^{R} u_{3 R}=m_{R}\left(r_{33}+2 r_{12}\right) \quad \hat{Y}_{a}^{R} v_{R a}=2 m_{R}\left(r_{11}-r_{23}\right), \\
& \hat{Y}_{b}^{R} v_{R b}=2 m_{R}\left(r_{22}-r_{13}\right) \quad \text { and } \quad \hat{Y}_{c}^{R} v_{R c}=2 m_{R}\left(r_{33}-r_{12}\right) .
\end{aligned}
$$

The $r_{i j}$ in Eq. (C4) are given by

$$
\begin{aligned}
& r_{11} \equiv \sqrt{2} b \sin 2 \theta_{12}^{0}+a \sin ^{2} \theta_{12}^{0}, \\
& r_{22} \equiv-\sqrt{2} b \sin \theta_{12}^{0}-\frac{b}{2} \sin 2 \theta_{12}^{0}-a \cos \theta_{12}^{0}+\frac{a}{2} \cos ^{2} \theta_{12}^{0}+\frac{a}{2}, \\
& r_{33} \equiv-\frac{b}{\sqrt{2}} \sin 2 \theta_{12}^{0}-\sqrt{2} b \sin \theta_{12}^{0}+a \cos \theta_{12}^{0}+\frac{a}{2} \cos ^{2} \theta_{12}^{0}+\frac{a}{2}, \\
& r_{12} \equiv b \cos 2 \theta_{12}^{0}+\frac{a}{2 \sqrt{2}} \sin ^{2} \theta_{12}^{0}+b \cos \theta_{12}^{0}-\frac{a}{\sqrt{2}} \sin \theta_{12}^{0}, \\
& r_{13} \equiv-b \cos 2 \theta_{12}^{0}-\frac{a}{2 \sqrt{2}} \sin ^{2} \theta_{12}^{0}+b \cos \theta_{12}^{0}-\frac{a}{\sqrt{2}} \sin \theta_{12}^{0}, \\
& r_{23} \equiv \frac{b}{2} \sin 2 \theta_{12}^{0}-\frac{a}{2} \cos ^{2} \theta_{12}^{0}+\frac{a}{2} .
\end{aligned}
$$

where $a$ and $b$ are dimensionless quantities of $\mathcal{O}(1)$. The charged lepton mass matrix is not diagonal in the Lagrangian basis. In order to go to a basis in which the charged lepton mass matrix $M_{e \mu \tau}$ is diagonal, a unitary transformation $U_{L}$ is applied on the left-handed lepton doublets. The transformation $V_{R}$ is applied on the righthanded neutrino singlets of $S U(2)_{L}$ such that the Dirac neutrino mass matrix remains proportional to identity in this transformed basis as well. This basis in which the charged lepton mass matrix is diagonal and the entire lepton mixing is dictated by the neutrino sector is called the flavor basis. The right-handed charged leptons were kept unchanged. The transformation matrices are given by

$$
U_{L}=\frac{1}{\sqrt{3}}\left(\begin{array}{ccc}
1 & 1 & 1 \\
1 & \omega^{2} & \omega \\
1 & \omega & \omega^{2}
\end{array}\right)=V_{R}
$$

The mass matrices in the flavor basis are

$$
\begin{gathered}
M_{e \mu \tau}^{\mathrm{flavor}}=\left(\begin{array}{ccc}
m_{e} & 0 & 0 \\
0 & m_{\mu} & 0 \\
0 & 0 & m_{\tau}
\end{array}\right), \\
M_{\nu L}^{\text {flavor }}=\frac{1}{2}\left(\begin{array}{ccc}
2 m_{1}^{(0)} & 0 & 0 \\
0 & m^{+} & m^{-} \\
0 & m^{-} & m^{+}
\end{array}\right), \\
M_{D}=f u \mathbb{I}, \quad M_{\nu R}^{\text {flavor }}=\frac{m_{R}}{4 a b}\left(\begin{array}{ccc}
r_{11} & r_{12} & r_{13} \\
r_{12} & r_{22} & r_{23} \\
r_{13} & r_{23} & r_{33}
\end{array}\right) .
\end{gathered}
$$

One can identify $f u=m_{D}$ where $m_{D}$ is the scale of the Dirac masses of the neutrinos. The type-I seesaw mechanism contribution is given by the matrices in Eq. (C8). 
[1] B. Brahmachari and A. Raychaudhuri, Phys. Rev. D 86, 051302 (2012); S. Pramanick and A. Raychaudhuri, Phys. Rev. D 88, 093009 (2013).

[2] S. Pramanick and A. Raychaudhuri, Phys. Lett. B 746, 237 (2015); Int. J. Mod. Phys. A 30, 1530036 (2015).

[3] P. Minkowski, Phys. Lett. B 67, 421 (1977); M. Gell-Mann, P. Ramond, and R. Slansky, in Supergravity, edited by F. van Nieuwenhuizen and D. Freedman (North Holland, Amsterdam, 1979), p. 315; T. Yanagida, in Proceedings of the Workshop on Unified Theory and the Baryon Number of the Universe (KEK, Japan, 1979); S. L. Glashow, NATO Sci. Ser. B 59, 687 (1980); R. N. Mohapatra and G. Senjanović, Phys. Rev. D 23, 165 (1981).

[4] F. Vissani, J. High Energy Phys. 11 (1998) 025; Models with somewhat similar points of view as those espoused here are E. K. Akhmedov, Phys. Lett. B 467, 95 (1999); M. Lindner and W. Rodejohann, J. High Energy Phys. 05 (2007) 089.

[5] For other recent work after the determination of $\theta_{13}$, see $\mathrm{S}$. Antusch, S. F. King, C. Luhn, and M. Spinrath, Nucl. Phys. B856, 328 (2012); B. Adhikary, A. Ghosal, and P. Roy, Int. J. Mod. Phys. A 28, 1350118 (2013); D. A. Sierra, I. de Medeiros Varzielas, and E. Houet, Phys. Rev. D 87, 093009 (2013); R. Dutta, U. Ch, A. K. Giri, and N. Sahu, Int. J. Mod. Phys. A 29, 1450113 (2014); L. J. Hall and G. G. Ross, J. High Energy Phys. 11 (2013) 091; T. Araki, Prog. Theor. Exp. Phys. 2013, 103 B02 (2013); A. E. C. Hernandez, I. de Medeiros Varzielas, S. G. Kovalenko, H. Päs, and I. Schmidt, Phys. Rev. D 88, 076014 (2013); M.-C. Chen, J. Huang, K. T. Mahanthappa, and A. M. Wijangco, J. High Energy Phys. 10 (2013) 112; B. Brahmachari and P. Roy, J. High Energy Phys. 02 (2015) 135.

[6] For a review, see, e.g., S. F. King and C. Luhn, Rep. Prog. Phys. 76, 056201 (2013).

[7] For the present status of $\theta_{13}$, see presentations from Double Chooz, RENO, Daya Bay, and T2K at Neutrino 2016 (http:// neutrino2016.iopconfs.org/programme).

[8] S. Pramanick and A. Raychaudhuri, Phys. Rev. D 93, 033007 (2016).

[9] E. Ma and G. Rajasekaran, Phys. Rev. D 64, 113012 (2001).

[10] G. Altarelli and F. Feruglio, Nucl. Phys. B741, 215 (2006); H. Ishimori, T. Kobayashi, H. Ohki, Y. Shimizu, H. Okada, and M. Tanimoto, Prog. Theor. Phys. Suppl. 183, 1 (2010).

[11] For a sampling see, e.g., F. Bazzocchi, S. Morisi, and M. Picariello, Phys. Lett. B 659, 628 (2008); E. Ma, Phys. Rev. D 73, 057304 (2006); P. Ciafaloni, M. Picariello, E. Torrente-Lujan, and A. Urbano, Phys. Rev. D 79, 116010
(2009); B. Brahmachari, S. Choubey, and M. Mitra, Phys. Rev. D 77, 073008 (2008); 77, 119901(E) (2008).

[12] E. Ma and D. Wegman, Phys. Rev. Lett. 107, 061803 (2011); S. Gupta, A. S. Joshipura, and K. M. Patel, Phys. Rev. D 85, 031903 (2012); G. C. Branco, R. G. Felipe, F. R. Joaquim, and H. Serodio, Phys. Rev. D 86, 076008 (2012); B. Adhikary, B. Brahmachari, A. Ghosal, E. Ma, and M. K. Parida, Phys. Lett. B 638, 345 (2006); B. Karmakar and A. Sil, Phys. Rev. D 91, 013004 (2015); E. Ma, Phys. Lett. B 752, 198 (2016).

[13] S. K. Kang and M. Tanimoto, Phys. Rev. D 91, 073010 (2015).

[14] S. Pramanick and A. Raychaudhuri, Phys. Rev. D 94, 115028 (2016).

[15] J. Barry and W. Rodejohann, Phys. Rev. D 81, 093002 (2010); J. Barry, W. Rodejohann, and H. Zhang, J. High Energy Phys. 07 (2011) 091; A. Adulpravitchai and R. Takahashi, J. High Energy Phys. 09 (2011) 127.

[16] R. G. Felipe, H. Serodio, and J. P. Silva, Phys. Rev. D 87, 055010 (2013).

[17] S. F. King and M. Malinsky, Phys. Lett. B 645, 351 (2007); E. Ma, H. Sawanaka, and M. Tanimoto, Phys. Lett. B 641, 301 (2006); S. Morisi, M. Nebot, K. M. Patel, E. Peinado, and J. W. F. Valle, Phys. Rev. D 88, 036001 (2013); S. Morisi, E. Peinado, Y. Shimizu, and J. W. F. Valle, Phys. Rev. D 84, 036003 (2011).

[18] E. Ma and U. Sarkar, Phys. Rev. Lett. 80, 5716 (1998).

[19] M. C. Gonzalez-Garcia, M. Maltoni, J. Salvado, and T. Schwetz, J. High Energy Phys. 12 (2012) 123, NuFIT 2.1 (2016).

[20] D. V. Forero, M. Tortola, and J. W. F. Valle, Phys. Rev. D 86, 073012 (2012).

[21] K. Abe et al. (T2K Collaboration), Phys. Rev. D 91, 072010 (2015), (see Fig. 37).

[22] P. Adamson et al. (NOVA Collaboration), Phys. Rev. D 116, 151806 (2016).

[23] M. Haag (KATRIN Collaboration), Proc. Sci., EPSHEP2013 (2013) 518.

[24] See, e.g., G. Drexlin, V. Hannen, S. Mertens, and C. Weinheimer, Adv. High Energy Phys. 2013, 293986 (2013).

[25] A. Degee, I. P. Ivanov, and V. Keus, J. High Energy Phys. 02 (2013) 125.

[26] S. Pramanick and A. Raychaudhuri, J. High Energy Phys. 01 (2018) 011.

[27] R. de Adelhart Toorop, F. Bazzocchi, L. Merlo, and A. Paris, J. High Energy Phys. 03 (2011) 035; 01 (2013) 098(E).

[28] R. G. Felipe, H. Serodio, and J. P. Silva, Phys. Rev. D 88, 015015 (2013). 